Article (refereed) - postprint

Gilbert, Mark A.; White, Steven M.; Bullock, James M.; Gaffney, Eamonn A. 2014. Spreading speeds for stage structured plant populations in fragmented landscapes.

Copyright (C) 2014 Elsevier Ltd.

This version available http://nora.nerc.ac.uk/504691/

NERC has developed NORA to enable users to access research outputs wholly or partially funded by NERC. Copyright and other rights for material on this site are retained by the rights owners. Users should read the terms and conditions of use of this material at http://nora.nerc.ac.uk/policies.html\#access

NOTICE: this is the author's version of a work that was accepted for publication in Journal of Theoretical Biology. Changes resulting from the publishing process, such as peer review, editing, corrections, structural formatting, and other quality control mechanisms may not be reflected in this document. Changes may have been made to this work since it was submitted for publication. A definitive version was subsequently published in Journal of Theoretical Biology (2014), 349. 135-149. 10.1016/j.jtbi.2014.01.024

www.elsevier.com/ noraceh@ceh.ac.uk 


\title{
Spreading Speeds for Stage Structured Plant Populations in Fragmented Landscapes
}

\author{
Mark A. Gilbert ${ }^{\mathrm{a}, \mathrm{b}, *}$, Steven M. White ${ }^{\mathrm{a}, \mathrm{b}}$, James M. Bullock ${ }^{\mathrm{b}}$, Eamonn A. Gaffney ${ }^{\mathrm{a}}$ \\ ${ }^{a}$ Wolfson Centre for Mathematical Biology, Mathematical Institute, Radcliffe Observatory Quarter, Woodstock Road, Oxford, \\ Oxfordshire, OX2 6GG, UK \\ ${ }^{b}$ Centre for Ecology 85 Hydrology, Benson Lane, Wallingford, Oxfordshire, OX10 8BB, UK
}

\begin{abstract}
Landscape fragmentation has huge ecological and economic implications and affects the spatial dynamics of many plant species. Determining the speed of population spread in fragmented/heterogeneous landscapes is therefore of utmost importance to ecologists. Stage-structured Integrodifference Equations (IDEs) are deterministic models which accurately reflect the life cycles and dispersal patterns for numerous species. Existing approximations to wave-speeds consider only particular kernels, or landscapes in which the scale of variation is much smaller than the dispersal scale. We propose an analytical approximation to the wavespeeds of IDE solutions with periodic landscapes of alternating good and bad patches, where the dispersal scale is greater than the extent of each good patch and where the ratio of the demographic rates in the good and bad patches is given by a small parameter, denoted $\epsilon$. We formulate this approximation for the Gaussian and Laplace dispersal kernels and for stage structured and non-stage structured populations, and compare the results against numerical simulations. We find that the approximation is accurate for the landscapes considered, and that the type of dispersal kernel affects the relationship between landscape structure, as classified by landscape period and good patch size, and the spreading speed. This indicates that accurately fitting a kernel to data is important in determining the relationship between landscape structure and spreading speed.
\end{abstract}

\section{Introduction}

The spread of plant populations has significant ecological and economic implications. Invasions by introduced plant species cause ecosystem degradation, loss of biodiversity [1], have detrimental impacts on human health and well-being [2], increase the rate of extinctions [3] and cost nations hundreds of billions of US dollars per year [4]. Conversely, in a conservation context, as the climate changes and the location of suitable habitat shifts, a native species' ability to survive will depend on its ability to spread at an equal or

*Corresponding author. Tel.: +44 (0)1865 283881

Email addresses: gilbert@maths.ox.ac.uk (Mark A. Gilbert), smwhit@ceh.ac.uk (Steven M. White), jmbul@ceh.ac.uk (James M. Bullock), gaffney@maths.ox.ac.uk (Eamonn A. Gaffney) 
greater rate than that at which its habitat shifts $[5,6,7]$. Therefore, understanding the process by which a species' range expands is of utmost importance, and an area of ongoing interest in ecology.

Most mathematical models of species' range expansion consider only spatially homogeneous landscapes (e.g. [7]) in which the demographic and dispersal parameters do not vary in space. However, landscape structure is very important in determining a species' ability to spread and its spreading speed [6, 8]. Landscape structure affects both dispersal and demographic processes, and is therefore of particular importance given the extent to which fragmentation affects the area and spatial distribution of habitats [9]. Fragmentation consists of two processes, the loss of suitable habitat and the increasing isolation of remaining habitat patches. It is a global phenomenon [10], which increases the vulnerability of landscapes to species invasion $[11,12]$ and may reduce species' ability to track regions of suitable climate as these regions shift $[13,14]$.

Plant populations are often studied using stage-structured matrix models [15], which can incorporate the different characteristics of a species at different points in its life-cycle. Such stage-structured representation can be incorporated into Integrodifference Equations [16], which are often used to model population spread in plants $[17,7,18,19,20]$. Continuous time, age-structured models have also been used [21]. We choose IDEs as our model as they incorporate important aspects of plant population behaviour, (1) they treat time as a discrete quantity, so accurately reflect the seasonal growth and dispersal of many plant species, and (2) incorporate different dispersal kernels to model various dispersal mechanisms. Spatial structure is generally continuous, although spatially discrete IDEs (or Coupled Map Lattices) have been studied [22].

A simple non stage-structured IDE relates the continuous population distribution $u^{t+1}(x)$ at time $t+1$, with the scalar distribution $u^{t}(x)$ at integer time $t$, where $x \in \mathbb{R}$ is a location in one dimensional space, via

$$
u^{t+1}(x)=\int_{-\infty}^{\infty} k(x-y, y) f\left(u^{t}(y), y\right) u^{t}(y) \mathrm{d} y
$$

In the growth phase, the population distribution is multiplied by the density and location dependent growth rate $f\left(u^{t}(y), y\right)[23,24,25,5]$. For the dispersal phase, the value of the post-dispersal population distribution at $x$ is obtained by taking the spatial integral of the product of the pre-dispersal population, $f\left(u^{t}(y), y\right) u^{t}(y)$ and the dispersal kernel $k(x-y, y)$, the relative density of dispersal from $y$ to $x$ [26]. In other words, the population abundance at spatial location $x$ at the next generation is simply the contribution from the birth/death processes, $f$, of the current generation that move to location $x$ according to redistribution kernel, $k$. Hence, the spatio-temporal population dynamics heavily depend on the growth and dispersal functions.

The long-term behaviour of solutions to IDEs can be studied through simulation, but analytical results are very useful, in that they help understand qualitative behaviour and dependencies on particular parameters, and provide a less computationally expensive way to study the dynamics of solutions to IDEs. For 
homogeneous IDEs with no Allee effect, where the growth and dispersal parameters are independent of location and the intrinsic growth rate $f(0, y)$ is higher than the growth rate experienced by any non-zero population $f(u, y)$, straightforward analytical expressions for the wave-speed have been derived [26]. For spatially heterogeneous IDEs, where the growth and dispersal parameters vary spatially, the analysis is less straightforward.

The solution of a spatially homogeneous IDE with no Allee effect is an exponential travelling wave [27], with the spreading behaviour being governed by the behaviour of the solution's wave-front [28]. This is often referred to as the Linear Conjecture [29], and allows us to approximate the IDE by its linearisation. For an homogeneous IDE, the growth rate $f\left(u^{t}(y), y\right)$ has no explicit $y$ dependence, allowing us to write it as $f\left(u^{t}(y)\right)$. The dispersal kernel $k(x-y, y)$ depends only on the distance $|x-y|$ travelled by the propagule, and has no explicit dependence on the origin $y$ of the propagule, allowing us to write it as $k(x-y)$. Given these conditions, Weinberger (1982) [30] showed that the asymptotic wave-speed for travelling wave solutions of (1) with bounded initial support is given by

$$
\hat{c}=\min _{s>0}\left(\frac{1}{s} \log (f(0) M(s))\right)
$$

where $M(s)$ is the moment generating function (MGF) of the dispersal kernel $k(x-y), M(s)=\int_{-\infty}^{\infty} k(z) \mathrm{e}^{s z} \mathrm{~d} z$. Neubert and Caswell (2000) incorporated stage structure into homogeneous IDEs, with the asymptotic wavespeed being given by

$$
\hat{c}=\min _{s>0}\left(\frac{1}{s} \log (\rho(s))\right)
$$

where $\rho(s)$ is the principal eigenvalue of the operator

$$
\mathbf{H}(s)=\int_{-\infty}^{\infty}[\mathbf{K}(z) \circ \mathbf{A}] \mathrm{e}^{s z} \mathrm{~d} z
$$

where $\circ$ denotes the Hadamard (elementwise) product of two matrices, $\mathbf{K}(z)$ denotes the stage structured dispersal kernel and $\mathbf{A}$ the stage-structured population projection matrix linearised around the zero population state [23].

For spatially heterogeneous IDEs, the lack of an equivalent expression to (2), has necessitated the development of other approaches to determine population persistence [31, 32], invasion conditions and wave-speeds [33]. The existing approaches all consider periodic landscapes, where the landscape is partitioned into periodically alternating good and bad patches, with the demographic rates and dispersal parameters taking fixed values in the different patch types. Kawasaki and Shigesda (2007) analysed the special case of the Laplace (exponential) dispersal kernel [34] and used the piecewise separability of the spatial variables in the kernel 
to derive conditions and speeds for invasions in periodic landscapes. Dewhirst and Lutscher (2009) [33] used averaging techniques to derive conditions and speeds for any exponentially bounded dispersal kernel in landscapes where the period of the landscape is much smaller than the scale of dispersal (although numerically the approximation has been found to work outside this range, up to the point where the dispersal scale and landscape period are of the same order). This difference in scales allows the replacement of the $f(0) M(s)$ term in (2) with the average of the two corresponding expressions for the good and bad patches to get the asymptotic wave speed of a non-stage structured IDE on a periodic landscape. The existing analytical methods have been successful in their agreement with simulations of IDEs, but are limited to non stage-structured populations and either (i) to a particular choice of kernel, which does not accurately describe the dispersal patterns of all species, e.g. [35], or (ii) to cases in which dispersal occurs at scales much larger than the distance between patches (Figure 1a), see [33, 35]. However, many fragmented habitats [36] such as calcareous grassland in Dorset, UK [9] and woodland in Wisconsin [37], as well as natural habitats such as vernal pools in California [38], do not conform to this pattern and are composed of small habitat fragments separated by distances which have sufficient length to make inter-patch dispersal rare. Hence, for general dispersal kernels, the spread of species in such landscapes cannot be analysed by existing methods. There is therefore a need for analytical approximations of invasion speeds which can incorporate a wide range of dispersal scales and kernels, stage structure and landscape heterogeneity, and are appropriate to a broader class of landscapes.

In this paper, we will address this important gap, and will derive analytical approximations for the asymptotic invasion speeds of stage structured populations in landscapes where (i) the spatial extent of good habitat patches is smaller than the scale of dispersal and (ii) the matrix of demographic rates in the bad habitat patches, are much lower than the matrix of demographic rates in the good patches (see Figure 1b). These approximations are not restricted to particular choices of landscape period, or to particular dispersal kernels and so extend the work of the previous studies. They also incorporate the important role of the bad habitat $[39,40]$ in determining invasion success/speed. To derive this approximation, we will exploit (i) the difference in scale between dispersal and the spatial extent of the good habitat patches and treat the population in each good patch as a population at a single point, and (ii) the much smaller growth rate in the bad patches to find the wave speed in terms of an asymptotic expansion in the ratio of the growth rates. In $\S 2$, we will present the derivation of an approximation to the asymptotic wave-speed of solutions to IDEs satisfying these conditions with compact initial support. In $\S 3$, we will derive particular approximations for IDEs with Gaussian and Laplace dispersal kernels, and will compare the approximations with simulation. We will conclude with a discussion of our results in $\S 4$. 
(a)

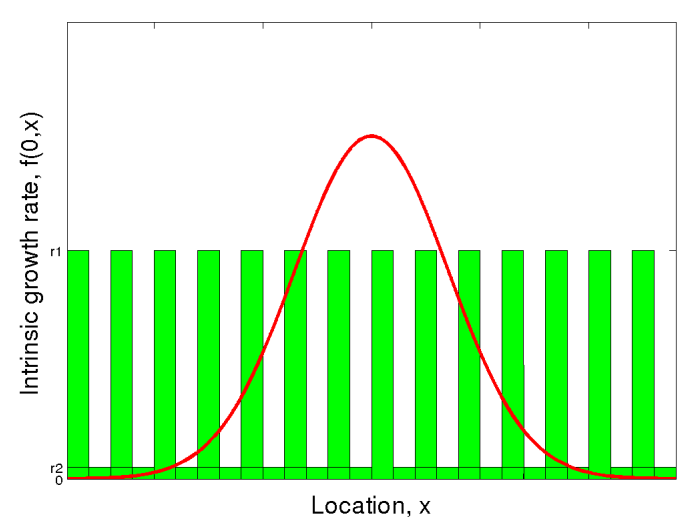

(b)

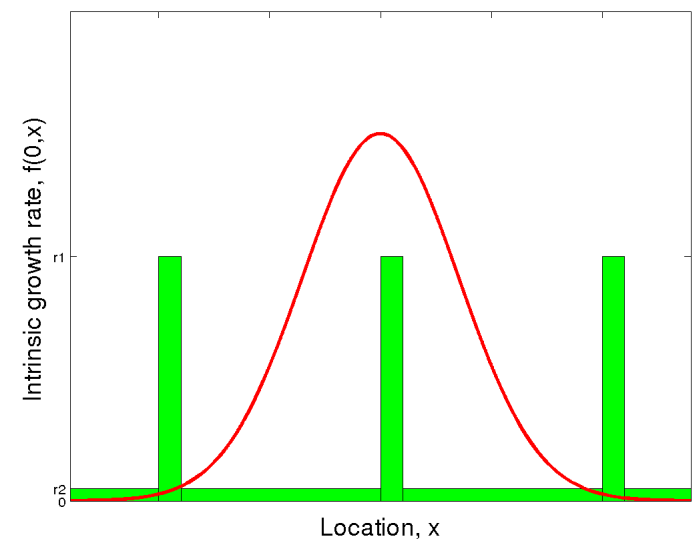

Figure 1: Intrinsic growth rates $f(0, x)$ (periodic square wave, green online) for a non stage-structured population in two periodic landscapes plotted alongside a dispersal kernel (unimodal, red online). In both landscapes, the growth rate in the bad patches are much lower than that in the good patches. In (a) the ratio of the landscape period to the mean dispersal distance is small (see Dewhirst and Lutscher [33]), and in (b), the ratio of the length of the good patches and the mean dispersal distance is small (considered in this paper).

\section{Method}

We begin by formulating a stage-structured IDE for heterogeneous landscapes with one spatial dimension. This is non-dimensional in the sense that we do not give scales or units to length, population density or time. The simplest population models treat all individuals as identical, regardless of size, age, sex, location etc. However, in many species, individuals exhibit demographically different characteristics at different points in the life-cycle. If the life cycle can be described in terms of size classes or developmental stages, then a useful way to categorize these differences is stage structure [15], where the population is partitioned into $M$ demographically relevant stages (seedlings, adults, etc).

This analysis is applicable to all choices of length and time scale (provided that the scale of dispersal is much larger than the extent of the good patches) and in this paper we consider non-dimensional IDEs, as they correspond to the variety of possible ecological scales. In $\S \S 2.1-2$, we will introduce this non-dimensional model, in $\S 2.3$, we give conditions for an analytical approximation of the wave-speed, and in $\S \S 2.4-5$ we will assume these to discuss wave-speed estimates.

\subsection{Heterogeneous Stage-Structured Integrodifference Equations}

The general heterogeneous stage-structured IDE [23] is given by

$$
\mathbf{u}^{t+1}(x)=\int_{-\infty}^{\infty}\left[\mathbf{K}(x-y, y) \circ \mathbf{B}\left(\mathbf{u}^{t}(y), y\right)\right] \mathbf{u}^{t}(y) \mathrm{d} y
$$

where $\mathbf{u}^{t}(x)$ is the vector of population densities for each stage at location $x$ and time $t . \mathbf{B}\left(\mathbf{u}^{t}(y), y\right)$ is the population projection matrix, and its $(i, j)$-th entry is the proportion of individuals in stage $i$ transitioning 
to stage $j . \mathbf{K}(x-y, y)$ is the matrix of dispersal kernels $K_{i, j}(x-y, y)$, where the $(i, j)$-th entry is the dispersal kernel for individuals which transitioned from stage $i$ to stage $j$ in the growth phase. It is necessary to consider the dispersal pattern of individuals transitioning between each (permitted) pair of demographic stages separately, as the stage of the individual or, in the case of juveniles, its parent before the growth phase will often affect the individual's dispersal behaviour. For example, for plants with wind dispersed seeds, the mean dispersal distance of a seed/new juvenile will depend on the seed release height of its parent and therefore on its parent's demographic stage.

\subsection{Sparse Periodic kernels and growth functions}

We consider one dimensional landscapes in which the real line $\mathbb{R}$ is partitioned into periodically alternating patch types, good patches $\mathcal{G}_{n}=\left[n L-L_{1} / 2, n L+L_{1} / 2\right)$ of length $L_{1}$, and bad patches $\mathcal{B}_{n}=\left[n L+L_{1} / 2, n L+\right.$ $\left.L-L_{1} / 2\right)$ of length $L-L_{1}$ (see Appendix C for a full table of parameters used in this manuscript). Hence $L$ is the period of the landscape. We assume that the dispersal kernel, $\mathbf{K}(x-y, y)$ and the population projection matrix, $\mathbf{B}\left(\mathbf{u}^{t}(y), y\right)$ depend solely on whether $y$ is in a good or bad patch, and do not vary within patches.

We make two assumptions about the landscape scales, dispersal parameters and demographic rates. We assume (i) that the length $L_{1}$ of the good patches is much smaller than the scale of the dispersal distances from the good patches (see Condition IV in $\S 2.3$ ), and (ii) that all the demographic rates in the bad patches are much lower than those in the good patches (see Condition V in $\S 2.3$ ).

Since the species has reduced fecundity in the bad patches (compared to the good patches), the population projection matrices in the good and bad patches are related by a small parameter $\epsilon \ll 1$, and are denoted respectively by $\mathbf{B}^{\mathcal{G}}\left(\mathbf{u}^{t}(y)\right)$ and $\epsilon \mathbf{B}^{\mathcal{B}}\left(\mathbf{u}^{t}(y)\right)$, where $\epsilon$ is chosen such that no element of the linearized population projection matrix $\mathbf{B}^{\mathcal{G}}(\mathbf{0})$ is much larger than the corresponding element in $\epsilon \mathbf{B}^{\mathcal{B}}(\mathbf{0})$. For brevity, the good and bad patch projection matrices are combined to form a spatially and density dependent population projection matrix,

$$
\mathbf{B}\left(\mathbf{u}^{t}(y), y\right)=\left\{\begin{array}{lll}
\mathbf{B}^{\mathcal{G}}\left(\mathbf{u}^{t}(y)\right) & \text { for } & y \in \mathcal{G}_{j} \\
\epsilon \mathbf{B}^{\mathcal{B}}\left(\mathbf{u}^{t}(y)\right) & \text { for } & y \in \mathcal{B}_{j}
\end{array} .\right.
$$

We also allow dispersal from $y$ to depend on whether $y$ is in $\mathcal{G}_{j}$ or $\mathcal{B}_{j}$, and let $\mathbf{K}^{\mathcal{G}}(x-y)$ and $\mathbf{K}^{\mathcal{B}}(x-y)$ be the matrices of dispersal kernels from the good and bad patches, with our spatially dependent matrix of dispersal kernels given by

$$
\mathbf{K}(x-y, y)=\left\{\begin{array}{lll}
\mathbf{K}^{\mathcal{G}}(x-y) & \text { for } & y \in \mathcal{G}_{j} \\
\mathbf{K}^{\mathcal{B}}(x-y) & \text { for } & y \in \mathcal{B}_{j}
\end{array} .\right.
$$




\subsection{Conditions}

We will explore solutions to (5) analytically by linearising, discretising and finding travelling wave solutions to the linearisation. All solutions corresponding to real populations have initial conditions with compact support. For a solution's asymptotic wave-speed to be determined by its linearisation, we need the spatially dependent demographic matrix $\mathbf{B}(\mathbf{x}, y)$ and matrix of dispersal kernels $\mathbf{K}(x-y, y)$ to satisfy conditions which are typical for IDE wave-speed analysis [23] and are commonly satisfied for population matrix modelling [15]:

I. The demographic matrix $\mathbf{B}(\mathbf{u}, y)$ is nonnegative and irreducible for all $\mathbf{u}$ and $y$, i.e. the transition rate between every pair of stages is non-negative, and the presence of individuals in any state will affect the number of individuals in any other state at some future time.

II. The intrinsic growth rate is greater than one (the zero population state is linearly unstable), and at every $y \in \mathbb{R}$ the non-linear population projection matrix $\mathbf{B}(\mathbf{u}, y)$ is bounded elementwise by its linearisation at zero, $\mathbf{A}(y):=\mathbf{B}(\mathbf{0}, y)$,

$$
\mathbf{B}(\mathbf{u}, y) \mathbf{u} \leq \mathbf{A}(y) \mathbf{u} \quad \text { for all } \quad \mathbf{u} \geq \mathbf{0}
$$

We observe numerically that this causes linear determinacy (that the asymptotic wave-speed of solutions to nonlinear IDE are the same as for the solutions of its linearisation). This condition is satisfied whenever higher populations cause reduced growth rates (through competition for resources, overcrowding, etc), which is true for most species. Linearly determinacy [41] has been proven rigorously only for the smaller class of cooperative systems. However, realistically, stage structured populations will exhibit competition between stages, and will not be cooperative.

III. Each element of the matrices of dispersal kernels from the good patches, $\mathbf{K}^{\mathcal{G}}(z)$ and bad patches, $\mathbf{K}^{\mathcal{B}}(z)$ have moment generating functions, i.e. the dispersal kernels are exponentially bounded. This ensures that solutions have finite wave-speeds and do not accelerate [26].

For our analysis of the heterogeneous landscape, we require two additional conditions,

IV. For the stage transitions where dispersal occurs, the scale of dispersal is much greater than the length $L_{1}$ of the good patches $\mathcal{G}_{j}$ (see Figure $1 \mathrm{~b}$ ). For this we require the $(i, j)^{\text {th }}$ element of the matrix of dispersal kernels from the good patch, $K_{i, j}^{\mathcal{G}}(z)$ to be either

(a) a Dirac delta function $\delta(z)$. No dispersal occurs for individuals transitioning from the $i^{\text {th }}$ to the $j^{\text {th }}$ demographic stage, or

(b) approximately constant over other (neighbouring) good patches, i.e. for landscape period $L$ and good patch length $L_{1}$,

$$
\mathbf{K}^{\mathcal{G}}(n L+z) \approx \mathbf{K}^{\mathcal{G}}(n L) \quad \text { for } \quad z \in\left[-\frac{L_{1}}{2}, \frac{L_{1}}{2}\right] \quad \text { and } n \in \mathbb{Z} \backslash\{0\}
$$


This condition is satisfied by species in habitats where the scale of dispersal is greater than the sizes of the individual habitat patches.

V. The population projection matrix in the good patches $\mathbf{B}^{\mathcal{G}}(\mathbf{x})$ is much larger than the population projection matrix in the bad patches $\epsilon \mathbf{B}^{\mathcal{B}}(\mathbf{x})$, i.e.

$$
\mathbf{B}^{\mathcal{G}}(\mathbf{x}) \gg \epsilon \mathbf{B}^{\mathcal{B}}(\mathbf{x})
$$

where inequalities are calculated component-wise. Generally, and by definition, the demographic rates of species are much lower in the bad patches than in the good patches.

\subsection{Method Outline}

To find an analytical approximation $c^{\text {app }}$ to the asymptotic wavespeed $\hat{c}$ of solutions to (5) where $\mathbf{B}$ and $\mathbf{K}$ are the demographic matrix and kernel given by (6) and (7), and where the solution has compact initial conditions (the initial distribution $\mathbf{u}^{0}(x)$ has compact support), we first linearise around the zero-population state. Since the zero-steady state is unstable, the population projection matrix is bounded by its linearisation at zero (Condition II) and density dependence is local, the dynamics of the invasion are dominated by the wavefront $[28,29]$. For the mathematical analysis, though not the later simulations, we will assume this Linear Conjecture [29], which has been proved rigorously for a narrower class of problem (see Appendix A). This allows us to explore solutions of a simpler, linear IDE, which has solutions with the same wave-speed as $(5)$.

We then look for solutions to the linearised IDE which are the product of an $L$-periodic stage-structured (vector-valued) population distribution and an exponential travelling wave $\mathrm{e}^{-s(x-c(s) t)}$ [42] which propagates with speed $c(s)$, where $s$ is the wave-number, and arbitrary a-priori. This gives us an eigenvalue equation

for $\rho_{s}:=\mathrm{e}^{s c(s)}$, with the principal eigenvalue corresponding to the physically meaningful solution of the linearised IDE [23]. Therefore, for each wave-number $s$, the wave-speed is given by

$$
c(s)=\frac{1}{s} \log \left(\rho_{s}\right)
$$

and the asymptotic wave-speed $\hat{c}$ is

$$
\hat{c}=\min _{s>0}\left[\frac{1}{s} \log \left(\rho_{s}\right)\right]
$$

Given the small demographic rates in the bad patches, we can write the operator in the eigenvalue equation as the sum of an $\mathrm{O}(1)$ operator, $F_{s, 0}$ and an $\mathrm{O}(\epsilon)$ operator, $\epsilon F_{s, 1}$ allowing us to solve the eigenvalue equation as a perturbation problem. We then approximate the $O(1)$ operator with a more tractable one, $F_{s, 0}^{\text {app }}$ (we justify this numerically in $§ 3.1 .4)$. This gives us the eigenvalue problem 


$$
F_{s}^{\mathrm{app}} \boldsymbol{\phi}_{s}^{\mathrm{app}}=\rho_{s}^{\mathrm{app}} \boldsymbol{\phi}_{s}^{\mathrm{app}}
$$

where $\rho_{s}^{\text {app }}$ is the principal eigenvalue of $F_{s}^{\text {app }}=F_{s, 0}^{\text {app }}+\epsilon F_{s, 1}$. Finally, we expand $\rho_{s}^{\text {app }}$ and $\phi_{s}^{\text {app }}$ in terms of the small parameter $\epsilon$ and equate the coefficients of $\epsilon^{n}(n=0,1, \ldots)$ in the expansion of (9). The expansion of $\rho_{s}^{\text {app }}$ is given by

$$
\rho_{s}^{\mathrm{app}}=\rho_{s, 0}^{\mathrm{app}}+\epsilon \rho_{s, 1}^{\mathrm{app}}+\cdots
$$

Our approximation to the wave-speed $c^{\text {app }}$ depends only on $\rho_{s, 0}^{\text {app }}$ and (where non-zero higher order terms exist) the second non-zero term in the expansion of $\rho_{s}^{\text {app }}$. i.e.

$$
c^{\text {app }}=\left\{\begin{array}{ll}
\min _{s>0}\left[\frac{1}{s} \log \left(\rho_{s, 0}^{\text {app }}\right)\right] & \text { if } \rho_{s, j}^{\text {app }}=0 \text { for all } j \neq 0 \\
\min _{s>0}\left[\frac{1}{s} \log \left(\rho_{s, 0}^{\text {app }}+\epsilon^{n} \rho_{s, n}^{\text {app }}\right)\right] & \text { where } n \text { is the smallest integer s.t. } \rho_{s, n}^{\text {app }} \neq 0
\end{array} .\right.
$$

So if $\rho_{s, 1}^{\text {app }}$ is non-zero, then our approximation to the wave-speed is $\min _{s>0}\left[\frac{1}{s} \log \left(\rho_{s, 0}^{\mathrm{app}}+\epsilon \rho_{s, 1}^{\mathrm{app}}\right)\right]$. If the $\mathrm{O}(\epsilon)$ terms cancel to make $\rho_{s, 1}^{\mathrm{app}}=0$, then we use the $\mathrm{O}\left(\epsilon^{2}\right)$ term and take $c^{\text {app }}=\min _{s>0}\left[\frac{1}{s} \log \left(\rho_{s, 0}^{\mathrm{app}}+\epsilon^{2} \rho_{s, 2}^{\mathrm{app}}\right)\right]$. If the $\mathrm{O}\left(\epsilon^{2}\right)$ terms also cancel and $\rho_{s, 2}^{\text {app }}=0$, we go to even higher order terms, and if all higher order terms are trivial, we take $c^{\text {app }}=\min _{s>0}\left[\frac{1}{s} \log \left(\rho_{s, 0}^{\mathrm{app}}\right)\right]$.

In the full derivation in Appendix A, we show that the principal eigenvalue $\rho_{s}^{\text {app }}$ of the approximate operator $F_{s}^{\text {app }}$ differs from the principal eigenvalue of $\rho_{s}$ of the exact operator $F_{s}$ by $\mathrm{O}\left(\left(L_{1} / \alpha\right)^{2}\right)$ where $\alpha$ is dispersal parameter. Since $\rho_{s, 0}^{\text {app }}+\epsilon^{n} \rho_{s, n}^{\text {app }}$ (where $n$ is the smallest integer such that $\rho_{s, n}^{\text {app }} \neq 0$ ) differs from $\rho_{s}^{\text {app }}$ by $\mathrm{O}\left(\epsilon^{n+1}\right), \rho_{s, 0}^{\text {app }}+\epsilon^{n} \rho_{s, n}^{\text {app }}$ differs from $\rho_{s}$ by $\mathrm{O}\left(\left(L_{1} / \alpha\right)^{2}, \epsilon^{n+1}\right)$. Since we must have $\rho_{s} \geq 1$ for an invasion to occur, we can expand $\log \left(\rho_{s}^{\text {app }}\right)$ in $\epsilon$ and $L_{1} / \alpha$ and find that $\log \left(\rho_{s}^{\text {app }}\right) / s=\log \left(\rho_{s}\right) / s+\mathrm{O}\left(\left(L_{1} / \alpha\right)^{2}, \epsilon^{n+1}\right)$. Therefore, we expect the minima of these two functions of $s$ to differ by $\mathrm{O}\left(\left(\frac{L_{1}}{\alpha}\right)^{2}, \epsilon^{n+1}\right)$, and the error of our approximation is $\mathrm{O}\left(\left(\frac{L_{1}}{\alpha}\right)^{2}, \epsilon^{n+1}\right)$.

\subsection{The Approximation}

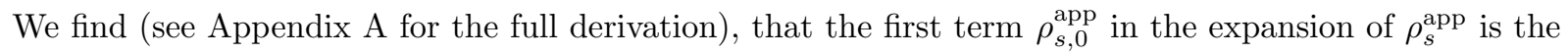
principal eigenvalue of

$$
\mathbf{M}_{0}(s)=\sum_{n \in \mathbb{Z}}\left[\mathbf{K}_{\text {app }}^{\mathcal{G}}(n L) \circ \mathbf{A}^{\mathcal{G}}\right] \mathrm{e}^{s n L}
$$

where $\mathbf{K}_{\text {app }}^{\mathcal{G}}(n L)$ is a discretisation of the dispersal kernel of propagules from the good patches given by

$$
\mathbf{K}_{\mathrm{app}}^{\mathcal{G}}(x-n L)=\left\{\begin{array}{cl}
\int_{-L_{1} / 2}^{L_{1} / 2} \mathbf{K}_{\mathrm{app}}^{\mathcal{G}}(y) \mathrm{d} y & \text { for } n=x=0 \\
L_{1} \mathbf{K}_{\mathrm{app}}^{\mathcal{G}}(x-n L) & \text { otherwise }
\end{array}\right.
$$


and where $\mathbf{A}^{\mathcal{G}}$ is the linear population projection matrix in the good patches (i.e. $\mathbf{B}^{\mathcal{G}}\left(\mathbf{u}^{t}(x)\right.$ ) at the zeropopulation state).

The second term $\rho_{s, 1}^{\text {app }}$ is given by

$$
\rho_{s, 1}^{\mathrm{app}}=\frac{\tilde{\boldsymbol{\psi}} \cdot\left[\mathbf{M}_{1}(s) \boldsymbol{\psi}\right]}{\rho_{s, 0}^{\mathrm{app}} \tilde{\boldsymbol{\psi}} \cdot \boldsymbol{\psi}}
$$

where $\boldsymbol{\psi}$ and $\tilde{\boldsymbol{\psi}}$ are the principal eigenvectors of (11) and its transpose, where $\mathbf{M}_{1}(s)$ is given by

$$
\mathbf{M}_{1}(s)=\left[\sum_{m, n \in \mathbb{Z}} \int_{\frac{-L_{1}}{2}}^{L-\frac{L_{1}}{2}}\left(\mathbf{K}^{\mathcal{B}}(n L-y) \circ \mathbf{A}^{\mathcal{B}}(y)\right)\left(\mathbf{K}_{\mathrm{app}}^{\mathcal{G}}(m L+y) \circ \mathbf{A}^{\mathcal{G}}\right) \mathrm{e}^{s(n+m) L} \mathrm{~d} y\right]
$$

and where $\mathbf{A}^{\mathcal{B}}$ is the linear population projection matrix in the bad patches.

Therefore, using the definition of $c^{\text {app }}$ in (10), the asymptotic wavespeed of solutions to (5) can be approximated by

$$
c^{\mathrm{app}}=\min _{s>0}\left[\frac{1}{s} \log \left(\rho_{s, 0}^{\mathrm{app}}+\epsilon \rho_{s, 1}^{\mathrm{app}}\right)\right] .
$$

This is the spatially heterogeneous equivalent to the expression for the asymptotic wave-speed of solutions to the spatially homogeneous IDE (2).

\subsection{Inhospitable Bad Pathces}

If the bad patches are completely inhospitable, i.e. the demographic matrix in the bad patches, $\mathbf{B}^{\mathcal{B}} \equiv \mathrm{O}$, then the higher order terms $\rho_{s, j}^{\text {app }}(j>0)$ in the expansion of $\rho_{s}^{\text {app }}$ are equal to zero. So, by the definition of $c^{\text {app }}$ in (10),

$$
c^{\mathrm{app}}=\min _{s>0}\left[\frac{1}{s} \log \left(\rho_{s, 0}^{\mathrm{app}}\right)\right]
$$

where $\rho_{s, 0}^{\text {app }}$ is the principal eigenvalue of $\mathbf{M}_{0}(s)$ in (11). The principal eigenvalue of this matrix is the ratio of the density distributions at $t+1$ and $t$, where the density distribution at $t$ is an exponential wave with wave-number $s$. This is a spatially discrete analogue of the matrix $\mathbf{H}(z)$ in (4).

\section{Examples}

\subsection{Analytical Approximations}

In this section, we first present approximations for the asymptotic wave speed of solutions to (5) where the demographic parameters vary between good and bad patches, but the choice of dispersal kernel (Gaussian or Laplace) and the dispersal parameters do not. We will find the first two non-zero terms in the expansion for 
$\rho_{s}^{\text {app }}$ for non stage-structured populations with Laplace (§3.1.1) and Gaussian (§3.1.2) dispersal kernels and stage-structured population with Laplace and Gaussian dispersal kernels (§3.1.3). In §3.1.4, we will justify the approximation of dispersal from a good patch with dispersal from a point source (the replacement of the integral operator $F_{s, 0}$ by $F_{s, 0}^{\text {app }}$ ) used in $\S 2.4$.

Throughout our non stage-structured simulations we use the Beverton-Holt growth function for $\mathbf{B}$. This is widely used as a population growth function and has been used to model plant populations [43], with the population after the growth phase being given by the current population $u$ multiplied by the growth rate

$$
f(u)=\frac{r K}{K+(r-1) u} .
$$

where $u$ is the scalar population before the growth phase, $r$ is the zero-population growth rate $(f(0)=r)$ and $K$ is the carrying capacity of the environment (here, without loss of generality, we set $K=1$ ). Since the approximation depends only on the zero-population growth rate, we could have used any growth function satisfying Condition II in $\S 2.3[26]$.

\subsubsection{Example with Laplace Kernel}

The non stage-structured Laplace dispersal kernel [33] is

$$
k(x-y)=\frac{1}{2 \alpha} \exp \left(-\frac{|x-y|}{\alpha}\right)
$$

with mean dispersal distance given by $\alpha$ for dispersal from any point in the landscape. Since this is a non stage-structured population, the linearised demographic growth matrices are the scalar growth rates $r$ (in the good patches) and $\epsilon r$ (in the bad patches). For the good patches, the discretised kernel, given by (12), is

$$
\mathrm{K}_{\mathrm{app}}^{G}(x-n L)=\left\{\begin{array}{cl}
1-\mathrm{e}^{\frac{-L_{1}}{2 \alpha}} & \text { for } n=x=0 \\
\frac{L_{1}}{2 \alpha} \mathrm{e}^{-\frac{|x-n L|}{\alpha}} & \text { otherwise }
\end{array} .\right.
$$

Using the result (11) from $\S 2.5$, we find an expression for $\rho_{s, 0}^{\text {app }}$,

$$
\begin{aligned}
\rho_{s, 0}^{\text {app }} & =r\left[\frac{L_{1}}{2 \alpha} \sum_{n \neq 0} \mathrm{e}^{\frac{-L|n|}{\alpha}+s L n}+\left(1-\mathrm{e}^{\frac{L_{1}}{2 \alpha}}\right)\right] \\
& =\frac{r L_{1}}{2 \alpha}\left[\left(\mathrm{e}^{\frac{L}{\alpha}+s L}-1\right)^{-1}+\left(\mathrm{e}^{\frac{L}{\alpha}-s L}-1\right)^{-1}\right]+r\left(1-\mathrm{e}^{\frac{L_{1}}{2 \alpha}}\right) .
\end{aligned}
$$

To get an expression for $\rho_{s, 1}$ we use (13), 


$$
\begin{aligned}
\rho_{s, 1}^{\text {app }} & =\frac{1}{\rho_{s, 0}} \frac{\epsilon r^{2} L_{1}}{4 \alpha^{2}} \sum_{n, m \in \mathbb{Z}} \mathrm{e}^{s L(n+m)} \int_{\frac{L_{1}}{2}}^{L-\frac{L_{1}}{2}} \mathrm{e}^{\frac{-1}{\alpha}(|L n-y|+|L m+y|)} \mathrm{d} y \\
& =\frac{1}{\rho_{s, 0}} \frac{\epsilon r^{2} L_{1}}{4 \alpha^{2}} \sum_{n, m \in \mathbb{Z}} \mathrm{e}^{\frac{-L}{\alpha} s L(n+m)} A_{n, m}
\end{aligned}
$$

where

$$
A_{n, m}=\left\{\begin{array}{lll}
\frac{1}{4 \alpha^{2}}\left(L-L_{1}\right) \mathrm{e}^{\frac{-1}{\alpha}|(m+n) L|} & \text { where } & \operatorname{sign}\left(m+\frac{1}{2}\right)=\operatorname{sign}\left(n-\frac{1}{2}\right) \\
\frac{1}{4 \alpha} \mathrm{e}^{\frac{-1}{\alpha} \operatorname{sign}\left(m+\frac{1}{2}\right)(m-n+1) L} \sinh \left(\frac{L-L_{1}}{\alpha}\right) & \text { where } & \operatorname{sign}\left(m+\frac{1}{2}\right) \neq \operatorname{sign}\left(n-\frac{1}{2}\right)
\end{array} .\right.
$$

We then substitute our expressions for $\rho_{s, 0}^{\text {app }}$ and $\rho_{s, 1}^{\text {app }}$ into (14) to get an analytical expression for the wavespeed which we will compare with numerical results in $\S 3.2$.

\subsubsection{Example with Gaussian Kernel}

The non stage-structured Gaussian dispersal kernel [33] is

$$
k(x-y)=\frac{1}{\sigma \sqrt{2 \pi}} \exp \left(\frac{-(x-y)^{2}}{2 \sigma^{2}}\right)
$$

with dispersal parameter $\sigma$ throughout the good and bad patches. The linear (zero-population) growth rates are $r$ (in the good patches) and $\epsilon r$ (in the bad patches). Given the smoothness of the Gaussian kernel around $x-y=0$, we can take our discretised kernel

$$
\mathrm{K}_{\mathrm{app}}^{G}(z)=L_{1} k(z) \quad \text { for all } z \in \mathbb{R}
$$

further simplifying the expression for $\mathrm{K}_{\mathrm{app}}^{G}$ in (12). By (11) we have that

$$
\rho_{s, 0}^{\text {app }}=\frac{r L_{1}}{\sigma \sqrt{2 \pi}} \sum_{n \in \mathbb{Z}} \mathrm{e}^{\frac{-n^{2} L^{2}}{2 \sigma^{2}}+s n L}
$$

and where erf is the error function, we have by (13),

$$
\begin{aligned}
\rho_{s, 1}^{\mathrm{app}} & =\frac{1}{\rho_{s, 0}^{\mathrm{app}}} \frac{r^{2} L_{1}}{2 \pi \sigma^{2}} \sum_{m, n \in \mathbb{Z}} \mathrm{e}^{s L(n+m)} \int_{\frac{L_{1}}{2}}^{L-\frac{L_{1}}{2}} \mathrm{e}^{\frac{-(L n-y)^{2}-(L m+y)^{2}}{2 \sigma^{2}}} \mathrm{~d} y \\
& =\frac{r^{2} L_{1}}{4 \sqrt{\pi} \sigma \rho_{s, 0}^{\mathrm{app}}} \sum_{m, n \in \mathbb{Z}} \mathrm{e}^{\frac{-L^{2}(n+m)^{2}}{4 \sigma^{2}}+s L(n+m)}\left[\operatorname{erf}\left(\frac{L(m-n+2)-L_{1}}{2 \sigma}\right)\right. \\
& \left.-\operatorname{erf}\left(\frac{L(m-n)+L_{1}}{2 \sigma}\right)\right] .
\end{aligned}
$$


We again substitute our expressions for $\rho_{s, 0}^{\text {app }}$ and $\rho_{s, 1}^{\text {app }}$ into (14) for an analytical expression for the wavespeed. The numerical evaluation of these expressions is straightforward, and as the terms in the infinite series become exponentially small as $n$ or $m$ tend to $\pm \infty$, they can be truncated to a finite number of terms (see Appendix B).

\subsubsection{Stage-Structured Example with Laplace and Gaussian Kernels}

We now find the first two non-zero terms in the expansion of $\rho_{s}^{\text {app }}$ for a stage-structured population with both Laplace and Gaussian kernels. The population has two stages (juveniles and adults), in which dispersal occurs only as juveniles are born. This is a modified version of the example in [23]. The population projection matrix in the good patches is

$$
\mathbf{B}^{\mathcal{G}}\left(\mathbf{u}^{t}(x)\right)=\left[\begin{array}{cc}
\nu(1-\gamma) & \phi \exp \left(-u_{1}^{t}(x)-u_{2}^{t}(x)\right) \\
\nu \gamma & \theta
\end{array}\right] .
$$

where $\nu$ and $\theta$ are the juvenile and adult survival rates (the proportion of individuals surviving to the next time-step), with $\gamma$ denoting the maturation rate (the proportion of juveniles advancing to adulthood) and $\phi \exp \left(-u_{1}^{t}(x)-u_{2}^{t}(x)\right)$ is the density dependent birth rate (the number of births per adult). The population projection matrix in the bad patches is $\epsilon \mathbf{B}^{\mathcal{G}}\left(\mathbf{u}^{t}(x)\right)$. Where new juveniles disperse with parameter $\alpha$, the dispersal kernel is

$$
\mathbf{K}(z)=\left[\begin{array}{cc}
\delta(z) & K(z) \\
\delta(z) & \delta(z)
\end{array}\right]
$$

where

$$
K(z)=\left\{\begin{array}{ll}
\frac{1}{2 \alpha} \exp \left(\frac{-|z|}{\alpha}\right) & \text { for the Laplace kernel } \\
\frac{1}{\sigma \sqrt{2 \pi}} \exp \left(\frac{-z^{2}}{2 \sigma^{2}}\right) & \text { for the Gaussian kernel }
\end{array} .\right.
$$

We use (12) to define the discretised kernel

$$
\mathbf{K}_{\mathrm{app}}(z)=\left[\begin{array}{cc}
\delta(z) & K_{\mathrm{app}}(z) \\
\delta(z) & \delta(z)
\end{array}\right]
$$

where, for the Gaussian and Laplace kernels, $K_{\text {app }}$ is defined as for the non stage-structured Gaussian and Laplace examples in (15) and (16). Substituting $\mathbf{K}_{\text {app }}$ and the linearisation of $\mathbf{B}^{\mathcal{G}}$ into (11), we find that for the Laplace kernel, $\rho_{s, 0}^{\text {app }}$ is the principal eigenvalue of 


$$
\left(\sum_{n \neq 0}\left[\begin{array}{cc}
\delta_{n, 0} & \frac{L_{1}}{2 \alpha} \exp \left(\frac{-|n L|}{\alpha}\right) \\
\delta_{n, 0} & \delta_{n, 0}
\end{array}\right] \mathrm{e}^{s n L}+\left[\begin{array}{cc}
\delta_{n, 0} & 1-\exp \left(\frac{-L_{1}}{2 \alpha}\right) \\
\delta_{n, 0} & \delta_{n, 0}
\end{array}\right]\right) \circ\left[\begin{array}{cc}
\nu(1-\gamma) & \phi \\
\nu \gamma & \theta
\end{array}\right]
$$

and for the Gaussian kernel, $\rho_{s, 0}^{\text {app }}$ is the principal eigenvalue of

$$
\sum_{n \in \mathbb{Z}}\left[\begin{array}{cc}
\delta_{n, 0} & \frac{L_{1}}{\sigma \sqrt{2 \pi}} \exp \left(\frac{-(n L)^{2}}{2 \sigma^{2}}\right) \\
\delta_{n, 0} & \delta_{n, 0}
\end{array}\right] \circ\left[\begin{array}{cc}
\nu(1-\gamma) & \phi \\
\nu \gamma & \theta
\end{array}\right] \mathrm{e}^{s n L}
$$

This means that

$$
\rho_{s, 0}^{\mathrm{app}}=\frac{1}{2}\left(\nu(1-\gamma)+\theta+\sqrt{(\nu(1-\gamma)+\theta)^{2}-4 \nu \gamma \phi B(s)}\right)
$$

where

$$
B(s)= \begin{cases}\frac{L_{1}}{2 \alpha}\left[\left(\mathrm{e}^{\frac{L}{\alpha}+s L}+1\right)^{-1}+\left(\mathrm{e}^{\frac{L}{\alpha}-s L}+1\right)^{-1}\right]+\left(1-\mathrm{e}^{\frac{-L_{1}}{2 \alpha}}\right) & \text { for the Laplace kernel } \\ \frac{L_{1}}{\sigma \sqrt{2 \pi}} \sum_{n \in \mathbb{Z}} \mathrm{e}^{\frac{-(n L)^{2}+s n L}{2 \sigma^{2}}} & \text { for the Gaussian kernel }\end{cases}
$$

Where $\tilde{\boldsymbol{\psi}}_{s}$ and $\boldsymbol{\psi}_{s}$ are the left and right eigenvectors of (18) for the Laplace kernel, or (19) for the Gaussian kernel, by (13), $\rho_{s, 1}$ is given by

$$
\rho_{s, 1}^{\mathrm{app}}=\frac{\tilde{\boldsymbol{\psi}}_{s} \mathbf{M}_{1} \boldsymbol{\psi}_{s}}{\rho_{s, 0} \tilde{\boldsymbol{\psi}}_{s} \boldsymbol{\psi}_{s}}
$$

where

$$
\begin{aligned}
\mathbf{M}_{1}= & \sum_{m, n \in \mathbb{Z}} \int_{\frac{L_{1}}{2}}^{L-\frac{L_{1}}{2}}\left(\left[\begin{array}{cc}
0 & K_{\mathrm{app}}(n L-y) \mathrm{e}^{s n L} \\
0 & 0
\end{array}\right] \circ\left[\begin{array}{cc}
\nu(1-\gamma) & \phi \\
\nu \gamma & \theta
\end{array}\right]\right) \\
& \times\left(\left[\begin{array}{cc}
0 & K_{\mathrm{app}}(m L+y) \mathrm{e}^{s m L} \\
0 & 0
\end{array}\right] \circ\left[\begin{array}{cc}
\nu(1-\gamma) & \phi \\
\nu \gamma & \theta
\end{array}\right]\right) \mathrm{d} y=\mathbf{O} .
\end{aligned}
$$

So, for both kernels, we have that $\rho_{s, 1}^{\text {app }}=0$, and to find the second non-zero term in the expansions of $\rho_{s}^{\text {app }}$, we must investigate the third term $\rho_{s, 2}^{\text {app }}$ in the expansion of $\rho_{s}^{\text {app }}$. To do this, we must find an expression for the second term $\phi_{s, 1}^{\text {app }}$ in the expansion of the eigenvector $\phi_{s}^{\text {app }}$. Since $\rho_{s, 1}^{\text {app }}=0$, the coefficients of $\epsilon$ in the expansion of the eigenvalue problem (9) satisfy

$$
\left(F_{s, 0}^{\mathrm{app}}-\rho_{s, 0}^{\mathrm{app}}\right) \boldsymbol{\phi}_{s, 1}^{\mathrm{app}}+F_{s, 1}^{\mathrm{app}} \boldsymbol{\phi}_{s, 0}^{\mathrm{app}}=0
$$


and by choice of $\phi_{s, 1}^{\text {app }}$

$$
\left\langle\tilde{\phi}_{s, 0}^{\mathrm{app}} \mid \boldsymbol{\phi}_{s, 1}^{\mathrm{app}}\right\rangle=0
$$

Both conditions (20) and (21) are satisfied by

$$
\phi_{s, 1}^{\mathrm{app}}=\frac{1}{\rho_{s, 0}^{\mathrm{app}}} \mathcal{I}_{\left[\frac{L_{1}}{2}, L-\frac{L_{1}}{2}\right)}(x) \sum_{m \in \mathbb{Z}}\left[\begin{array}{cc}
0 & \nu(1-\gamma) \phi \\
0 & \nu \gamma \phi
\end{array}\right] K(x-m L) \mathrm{e}^{s(x-m L)} \boldsymbol{\psi}_{s}
$$

where $K(z)$ is the dispersal kernel of newborn juveniles defined in (17). Taking the coefficients of $\epsilon^{2}$ in (9), we have

$$
\left(F_{s, 0}^{\mathrm{app}}-\rho_{s, 0}^{\mathrm{app}}\right) \phi_{s, 2}^{\mathrm{app}}+\left(F_{s, 1}^{\mathrm{app}}-\rho_{s, 1}^{\mathrm{app}}\right) \phi_{s, 1}^{\mathrm{app}}=\rho_{s, 2}^{\mathrm{app}} \phi_{s, 0}^{\mathrm{app}}
$$

Taking the inner-product with $\tilde{\phi}_{s, 0}^{\text {app }}$, the first term on the left hand side becomes zero $\left(\tilde{\phi}_{s, 0}^{\text {app }}\right.$ is the eigenvector of the adjoint operator of $F_{s, 0}^{\text {app }}$ with eigenvalue $\rho_{s, 0}^{\text {app }}$ ), and so

$$
\begin{aligned}
\rho_{s, 2}^{\mathrm{app}} & =\frac{\left\langle\tilde{\boldsymbol{\phi}}_{s, 0}^{\mathrm{app}} \mid F_{s, 1}^{\mathrm{app}} \boldsymbol{\phi}_{s, 1}^{\mathrm{app}}\right\rangle}{\left\langle\tilde{\boldsymbol{\phi}}_{s, 0}^{\mathrm{app}} \mid \boldsymbol{\phi}_{s, 0}^{\mathrm{app}}\right\rangle} \\
& =\frac{L_{1} \phi^{2} \nu \gamma \tilde{\boldsymbol{\psi}}_{s}^{1} \boldsymbol{\psi}_{s}^{2}}{\left(\rho_{s, 0}^{\mathrm{app}}\right)^{2} \tilde{\boldsymbol{\psi}}_{s} \boldsymbol{\psi}_{s}} \sum_{m, n \in \mathbb{Z}} \mathrm{e}^{s L(m+n)} \int_{\frac{L_{1}}{2}}^{L-\frac{L_{1}}{2}} K(n L-y) K(m L+y) \mathrm{d} y \\
& =\frac{L_{1} \phi^{2} \nu \gamma \tilde{\boldsymbol{\psi}}_{s}^{1} \boldsymbol{\psi}_{s}^{2}}{\left(\rho_{s, 0}^{\mathrm{app}}\right)^{2} \tilde{\boldsymbol{\psi}}_{s} \boldsymbol{\psi}_{s}} \sum_{m, n \in \mathbb{Z}} \mathrm{e}^{s L(m+n)} A_{n, m}
\end{aligned}
$$

where, for the Laplace kernel

$$
A_{n, m}=\left\{\begin{array}{lll}
\frac{1}{4 \alpha^{2}}\left(L-L_{1}\right) \mathrm{e}^{\frac{-1}{\alpha}|(m+n) L|} & \text { where } & \operatorname{sign}\left(m+\frac{1}{2}\right)=\operatorname{sign}\left(n-\frac{1}{2}\right) \\
\frac{1}{4 \alpha} \mathrm{e}^{\frac{-1}{\alpha} \operatorname{sign}\left(m+\frac{1}{2}\right)(m-n+1) L} \sinh \left(\frac{L-L_{1}}{\alpha}\right) & \text { where } & \operatorname{sign}\left(m+\frac{1}{2}\right) \neq \operatorname{sign}\left(n-\frac{1}{2}\right)
\end{array}\right.
$$

and for the Gaussian kernel

$$
A_{n, m}=\frac{1}{4 \sqrt{\pi} \sigma} \mathrm{e}^{\frac{-L^{2}(n+m)^{2}}{4 \sigma^{2}}}\left[\operatorname{erf}\left(\frac{L(m-n+2)-L_{1}}{2 \sigma}\right)-\operatorname{erf}\left(\frac{L(m-n)+L_{1}}{2 \sigma}\right)\right] .
$$

We now have expressions for $\rho_{s, 0}^{\text {app }}$ and $\rho_{s, 2}^{\text {app }}$ and have found that $\rho_{s, 1}^{\text {app }}=0$. The approximation to the asymptotic wave-speed is given by (10),

$$
c^{\text {app }}=\min _{s>0}\left[\frac{1}{s} \log \left(\rho_{s, 0}^{\mathrm{app}}+\epsilon^{2} \rho_{s, 2}^{\mathrm{app}}\right)\right] .
$$


(a)

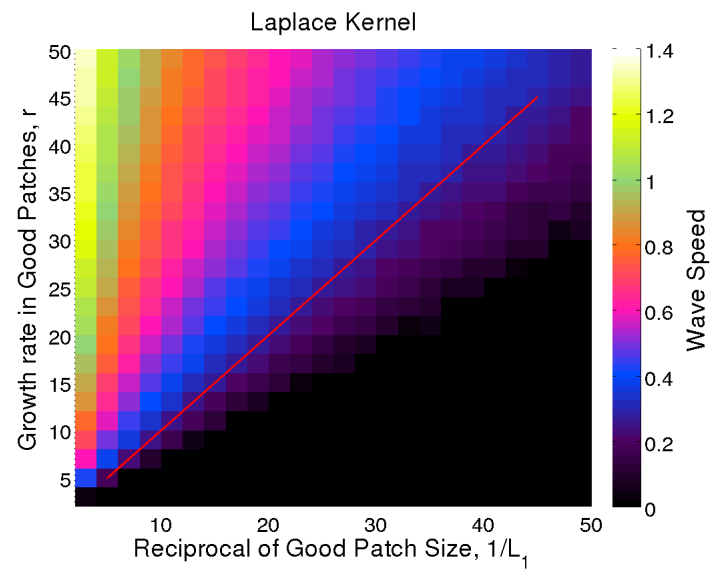

(b)

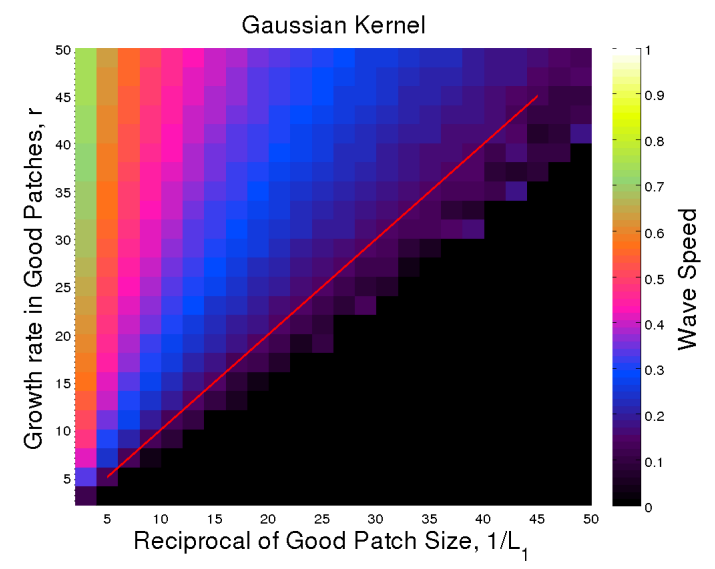

Figure 2: (Colour online) wave-speeds for $2 \leq r \leq 50$ and $2 \leq 1 / L_{1} \leq 50$ for $r_{2}=0.1, L=1$ for (a) a Laplace kernel with $\alpha=0.3$ and (b) a Gaussian kernel with $\sigma=0.3$. The wave-speed is constant along the lines of the form $r=A / L_{1}$ (explicitly highlighted for $A=1$ ).

\subsubsection{Justification of the Point Source Approximation}

By approximating dispersal from each good patch with dispersal from a point source (approximating the integral operator $F_{s, 0}$ with $F_{s, 0}^{\mathrm{app}}$ ) in $\S 2.5$, we assumed that the widths of the good patches are sufficiently small that neglecting the effect of spatial structure within each good patch, except when considering the quantity of dispersers remaining in the same patch, does not affect the asymptotic wave-speed of solutions to $(5)$.

For small populations, the quantity of propagules leaving the good patch is proportional to the linearised scalar population growth rate multiplied by the width of the good patches, $r L_{1}$. For our approximation to hold, the asymptotic wave-speeds of solutions to (5) must be constant as $L_{1} \rightarrow 0$ with constant $r L_{1}$.

We simulated solutions to the non stage-structured IDE (5) for the Laplace and Gaussian dispersal kernels defined in $\S \S 3.1 .1-2$ using the Beverton-Holt population growth function (§2.7) with different values of the good patch zero-population growth rate $r$ and good patch width $L_{1}$ while keeping the dispersal parameters (which do not vary between the good and bad patches), landscape period $L$ and bad patch zero-population growth rate $r_{2}$ constant (see Figure 2). We found that the contour lines (lines of constant wave speed) are given by

$$
r L_{1}=\text { Const. }
$$

and that changing the landscape parameter's position on the line (23) does not affect the asymptotic wavespeed of solutions to (5). This justifies the use of a point source approximation and hence using $F_{s, 0}^{\text {app }}$ as an approximation to $F_{s, 0}$ to get the analytical results in $\S 2.5$. 


\subsection{Numerical Results}

We simulate solutions to (5) for the population projection matrices $\mathbf{B}\left(\mathbf{u}^{t}(x), x\right)$ and dispersal kernels $\mathbf{K}(x-y)$ of each of the above examples (see Appendix B for numerical details). We simulate solutions for a range of values of the total proportion of good habitat $p=L_{1} / L$ and landscape period $L$. Since the dispersal pattern of a given plant species is somewhat fixed, we fix the dispersal parameters, $\alpha=1$ (for the Laplace kernel) or $\sigma=1$ (for the Gaussian kernel), and vary the total proportion of good habitat in the landscape $p$ and the landscape period $L$.

Additionally, we choose the population growth rates in the good patches (i.e. the principal eigenvalue of the demographic matrix) to be higher than those used in applications of homogeneous IDEs (e.g. [17]). For homogeneous IDEs, the most appropriate approach would be take a spatial average of demographic rates. In the heterogeneous model, we distinguish between the demographic rates in the good and bad patches, and would expect higher and lower demographic rates in good and bad habitat patches respectively.

We investigate the effect of these parameters on the relative errors of the approximations from $\S \S 3.1 .1-3$ compared to the simulated wave-speed in $\$ 3.2 .2$, and investigate their effect on the simulated wave speed in $\S 3.2 .3$.

\subsubsection{Effect of the Bad patches on the wave-number}

For both the Laplace and Gaussian kernels, the first-order approximation of the wave-speed is given by (14) in $\S 2.5$. In Figure 3, we plot the zeroth (considering only $\rho_{s, 0}^{\text {app }}$ ) and first order approximations to the wave-speed $c(s)$ for a range of wave-numbers $s$ for both non stage-structured examples. The minima of the first order approximation give significantly better agreement with the simulated wave-speed than the minima of the zeroth order approximation. The first order approximation also has a higher minimum wavespeed than the zeroth order approximation, and these minima correspond to different wave-numbers (see Figure 3).

\subsubsection{Relative Errors}

For the examples in $\S \S 3.1 .1-3$, we found that for the values of the total proportion of good habitat $p=L_{1} / L$ and landscape period $L$ where the simulated wave-speed, $c^{\operatorname{sim}} \neq 0$ (the relative error is not defined for $c^{\operatorname{sim}}=0$ ) the relative error

$$
E=\left|\frac{c^{\mathrm{app}}-c^{\mathrm{sim}}}{c^{\mathrm{sim}}}\right|
$$

is no more than 0.1, except for values of $c^{\text {sim }}$ close to 0 (see Figure 4). The error is small, and we have shown that the minima of the log of the first two non-zero terms of the expansion of $\rho_{s}^{\text {app }}$ divided by the wavenumber, $s$ gives good agreement to the long-term wave-speed of simulated solutions to (5). Therefore the first two non-zero terms in the expansion of $\rho_{s}^{\text {app }}$ correspond to an accurate approximation to the asymptotic 
(a)

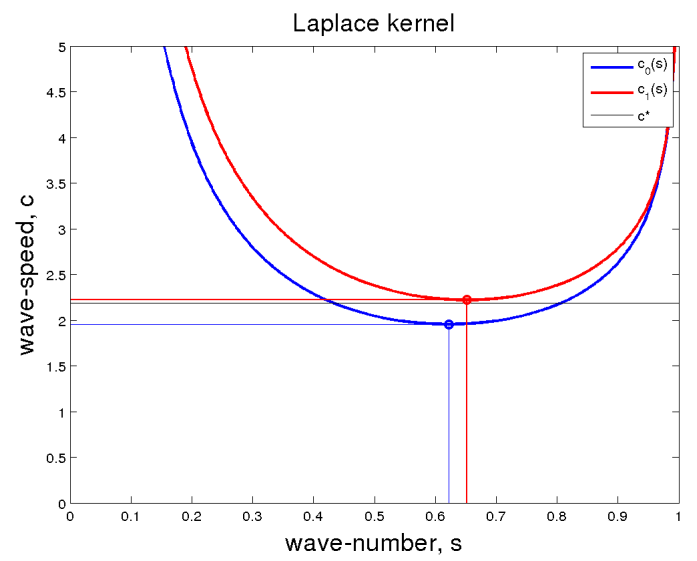

(b)

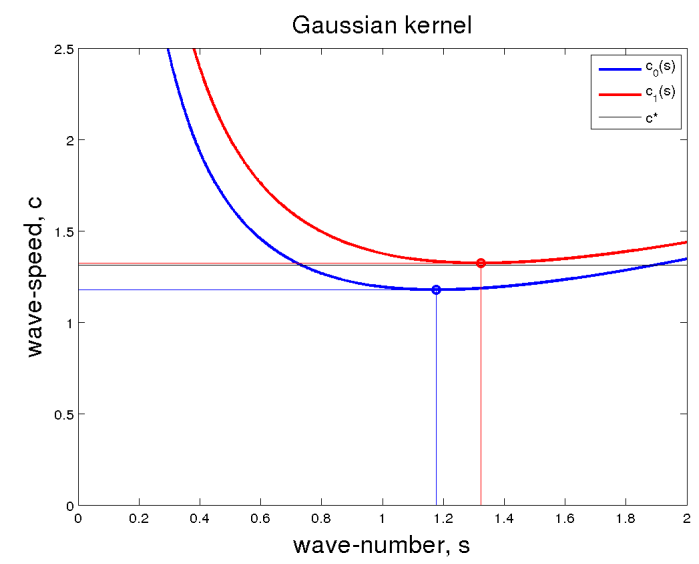

Figure 3: (Colour online) zeroth order $c_{0}(s)$ (blue) and first order $c_{1}(s)$ (red) approximations to the wave-speed $c(s)$ of a travelling wave of the linearised IDE where $r=10, L_{1}=0.2$ and $L=1$ as a function of the wave-number $s$, for (a) a Laplace kernel where $\alpha=1$ and (b) a Gaussian kernel where $\sigma=1$. The red and blue dots show the location of the minima of $c_{1}(s)$ and $c_{0}(s)$ respectively. The horizontal black line $c^{*}$ shows the asymptotic wave-speed found through simulation.

wave-speed for the range of parameters studied for both (1) cases where the first two non-zero terms are $\rho_{s, 0}^{\text {app }}$ and $\rho_{s, 1}^{\text {app }}$ (see Figure 4a-d), and for (2) cases where the first two non-zero terms are $\rho_{s, 0}^{\text {app }}$ and $\rho_{s, 2}^{\text {app }}$ (see Figure $4 \mathrm{e}-\mathrm{h})$

\subsubsection{Effects of Fragmentation}

When studying the effect of fragmentation on landscape invasibility and invasion speed, we consider both the overall amount of habitat and the level of isolation of the patches. To explore the effect of habitat loss, we vary the total proportion of good habitat $p=L_{1} / L$, and to explore the effect of patch isolation, we vary the landscape period $L$ (the scale of the landscape relative to the scale of dispersal).

For all examples, increasing the total proportion of good habitat $p$ increases the wave-speed (For each of the plots in Figure 5, increased $p$ corresponds to higher wave-speeds). Similarly, increasing the ratio $\epsilon$ of the demographic rates in the bad patches relative to the rates in the good patches increases the wave speed (the wave speeds in Figure 5b,d,f,h are higher than for the corresponding values of $L$ and $p$ in Figure $5 \mathrm{a}, \mathrm{c}, \mathrm{e}, \mathrm{g}$ ).

For both the non-stage structured and stage-structured examples, the relationship between the landscape period $L$ and invasion speed differs between the two dispersal kernels. For the Gaussian kernel, and the values of $p$ (the proportion of good habitat) for which an invasion occurs when the landscape period $L \sim 1$, the wave speed decreases with increased $L$ (except for a small region of parameter space in Fig $5 \mathrm{~d}$, where a minor increase occurs). For the Laplace kernel, increasing $L$ increases the wave speed.

For both non stage-structured examples (Figure 5a-d) and for both stage-structured examples with $\epsilon=0$ (Figure 5e,g), we found that increasing the landscape period $L$ decreases the total proportion of good habitat, $p$ needed for an invasion to occur. Our results do not show this for the stage-structured examples with $\epsilon=0.25$ (Figure 5f,h) as all values of $p$ and $L$ correspond to an invasion. 
(a)

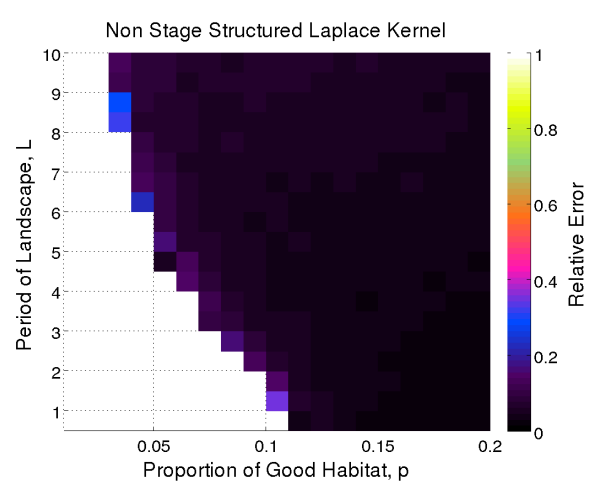

(c)

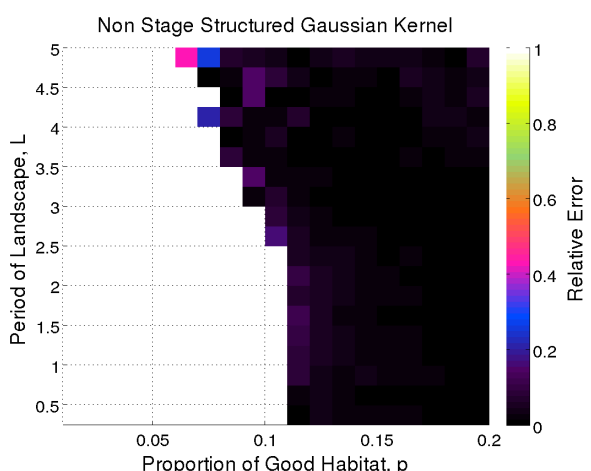

(e)

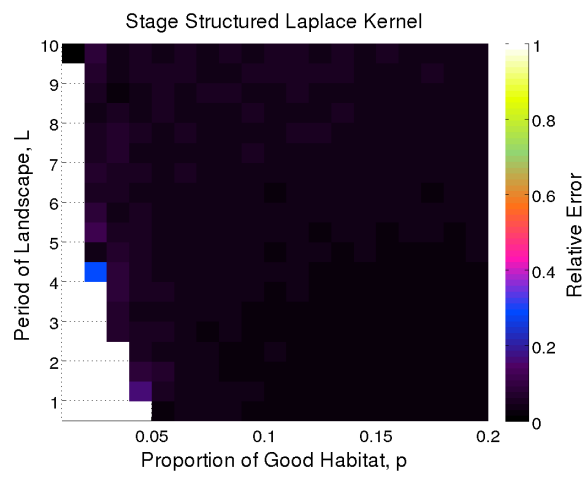

(g)

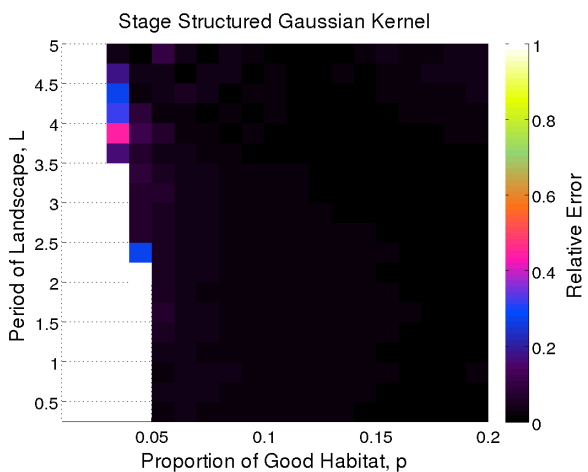

(b)

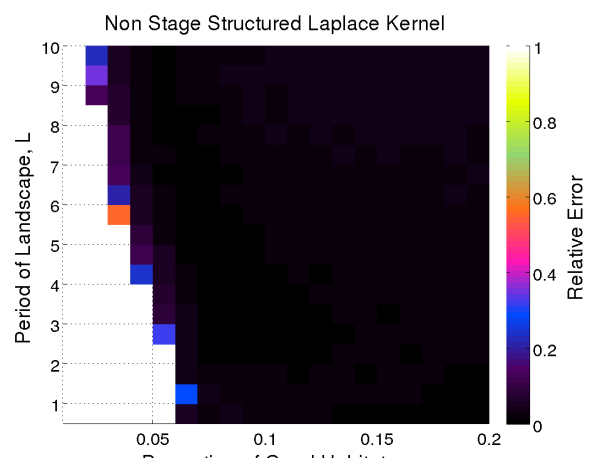

(d)

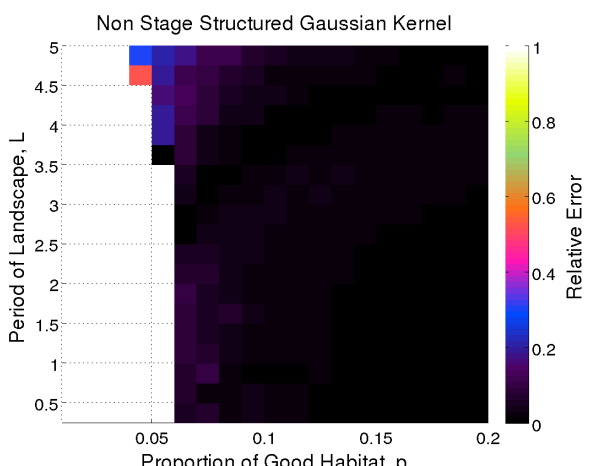

(f)

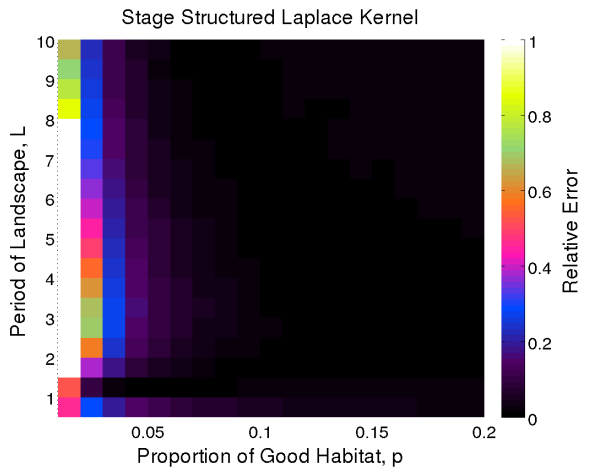

(h)

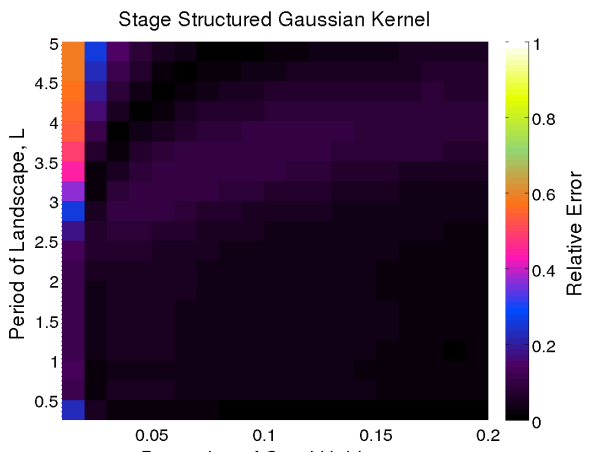

Proportion of Good Habitat,

Figure 4: (a)-(d) The relative errors of the first order analytic approximation (14) to the simulated wave-speeds of the non-stage structured IDEs with $r=10$ and (a)-(b) Laplace Kernel with $\alpha=1$ and (a) $\epsilon=0$, (b) $\epsilon=0.05$. (c)-(d) Gaussian kernel with $\sigma=1$ and (c) $\epsilon=0,(\mathrm{~d}) \epsilon=0.05$. (e)-(h) Relative errors of the second order analytic approximation (22) to the stage-structured IDE with $\nu=0.8, \gamma=0.625, \theta=0.2, \phi=30$ and $\alpha=1$ and (e)-(f) Laplace kernel with $\alpha=1$ and (e) $\epsilon=0$, (f) $\epsilon=0.25$. (g)-(h) Gaussian kernel with $\sigma=1$ and $(\mathrm{g}) \epsilon=0,(\mathrm{~h}) \epsilon=0.25$. The blank space at the left of each plot corresponds to values of $L$ and $p$ for which the wave cannot propagate, and the relative error is not defined. For both kernels, where the relative error is defined, it is less than 0.1 for a wide range of the parameters of interest. A table of parameters is given in Appendix C. 
To spread, a species with given dispersal and growth parameters requires a lower total proportion of good habitat when the landscape period is greater (the width of the black region corresponding to no spreading decreases as $L$ increases in Figure 5a-d,e,g). 
(a)

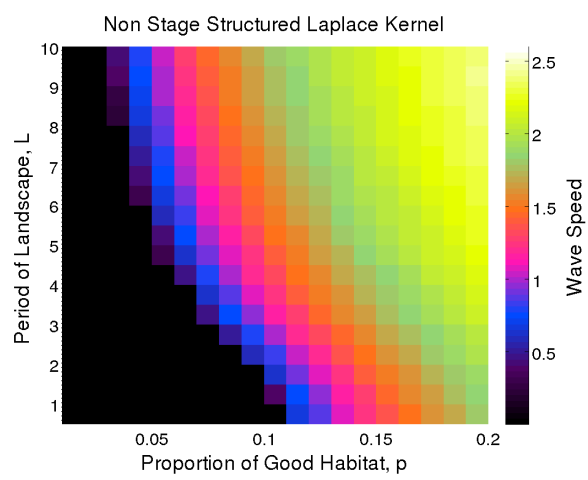

(c)

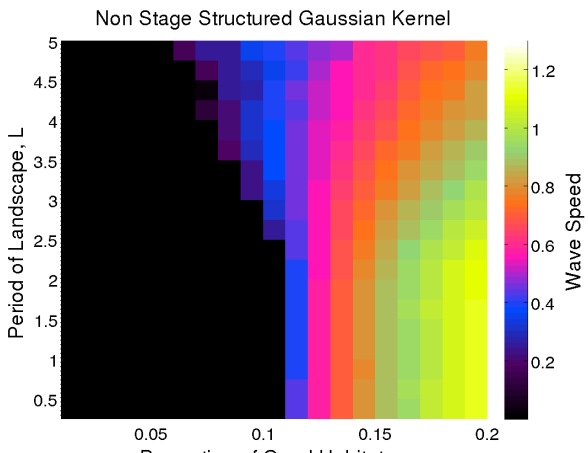

(e)

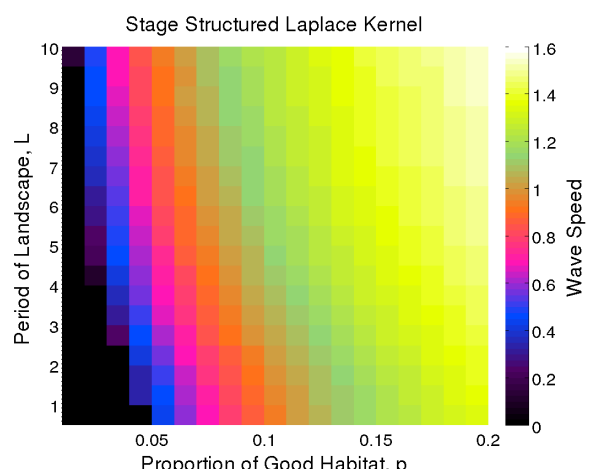

(g)

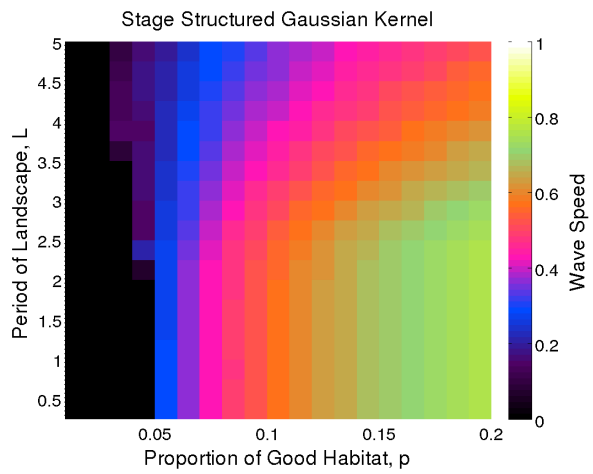

(b)

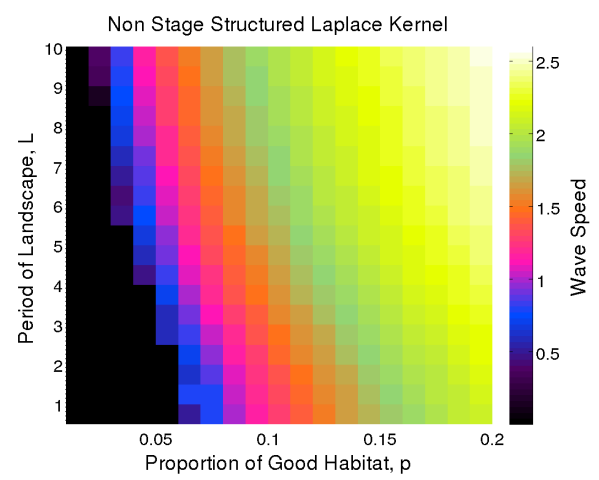

(d)

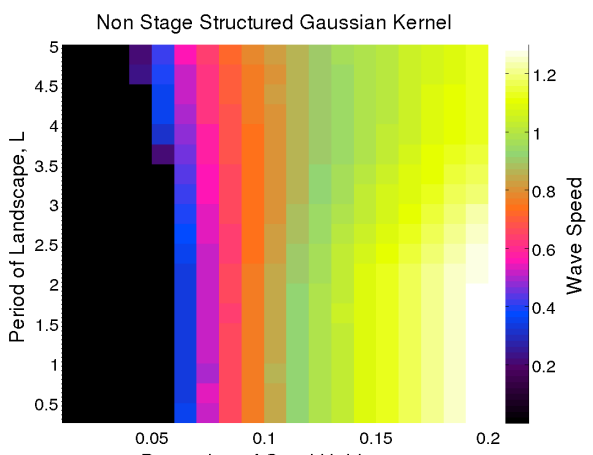

(f)

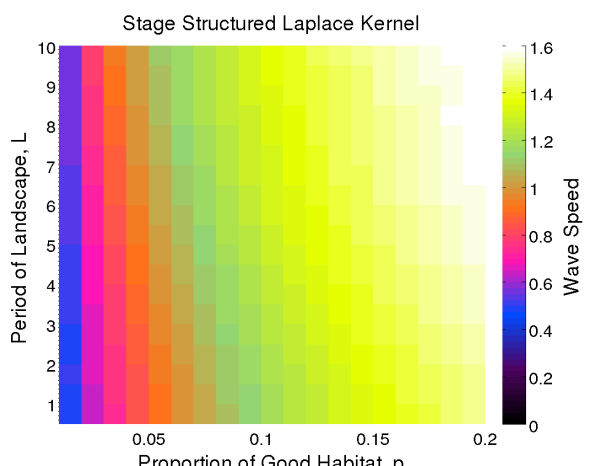

(h)

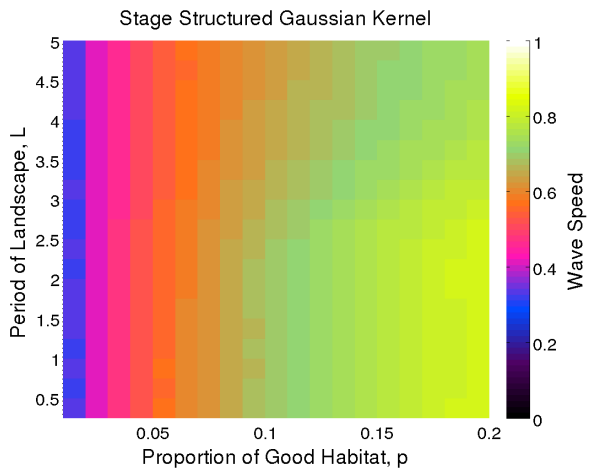

Figure 5: (a)-(d) Simulated asymptotic wave-speeds of non stage-structured IDEs with $r=10$ for $0.01 \leq L_{1} / L \leq 0.2$ with (a)-(b) Laplace Kernel with $\alpha=1$ and (a) $\epsilon=0$, (b) $\epsilon=0.05$. (c)-(d) Gaussian kernel with $\sigma=1$ and (c) $\epsilon=0$, (d) $\epsilon=0.05$. (e)-(h) Simulated wave-speeds of the stage-structured IDE with $\nu=0.8, \gamma=0.625, \theta=0.2, \phi=30$ and $\alpha=1$ and (e)-(f) Laplace kernel with $\alpha=1$ and (e) $\epsilon=0$, (f) $\epsilon=0.25$. (g)-(h) Gaussian kernel with $\sigma=1$ and (g) $\epsilon=0,(\mathrm{~h}) \epsilon=0.25$. 


\section{Discussion}

In this paper, we have derived an approximation for the asymptotic wave-speeds of solutions to stagestructured IDEs on periodic landscapes by finding the minimum of the principal eigenvalues for the operators $F_{s}$. Approximating wave-speeds is useful because it yields analytically justified results and does not rely on numerical simulation, which is time-consuming, does not provide the same insight as an analytical approximation (e.g. sensitivity to certain parameters) and does not allow extensive parameter sweeps.

This is the first approximation to incorporate both stage-structure and landscape heterogeneity. The averaging approximation of Dewhirst and Lutscher [33], which approximates the sum of the density of dispersal from each patch as a Riemann integral, is valid only for landscapes in which the landscape period $L$ (and therefore the extent of the good/bad patches) is much less than the dispersal scale, $L \ll \alpha, \sigma$, and breaks down when the landscape period is too large. Here, we have removed any condition on the landscape period, and replaced it with the less restrictive condition that the length scale of the good patch $L_{1}$ satisfies $L_{1} \ll \alpha, \sigma$ (Condition IV). This is sufficient to justify the approximation of the operator $F_{s}$ in $\S 2.4$ with the discretised operator $F_{s}^{\text {app }}$. Additionally, we require the demographic rates in the bad patches to be related to the corresponding rates in the good patches by the small parameter $\epsilon \ll 1$ (Condition V). Taken together, these two assumptions allow us to express the principal eigenvalue $\rho_{s}^{\text {app }}$ of $F_{s}^{\text {app }}$ as an expansion in $\epsilon$, with the terms in the expansion given as expressions of the demographic and dispersal parameters. Breaking the first of these assumptions (that $\left.L_{1} \ll \alpha, \sigma\right)$ makes our approximation of $F_{s}$ by $F_{s}^{\text {app }}$ unreasonable. Therefore, we do not expect this approximation to work when $L_{1}$ is of the same or a greater scale than the dispersal parameter. Increasing $\epsilon$ will increase the impact of higher order terms on the wave-speed, and when $\epsilon \sim 1$ we would no longer expect the first few terms in the expansion in $\epsilon$ to correspond to an approximation to the wave-speed.

We compared our approximation against numerical simulations for a range of parameters for Laplace and Gaussian dispersal kernels and for stage-structured and non-stage-structured populations. We found that the relative error of the approximation (compared to the simulations) is small (Figure 4), i.e. that the approximation of $\hat{c}$ given by $c^{\text {app }}$ is accurate for the values of the parameters studied. The approximation is valid for any of the more complex exponentially bounded kernels which are often used in ecology [44]. For these kernels, terms in the expansion of the eigenvalue in $\epsilon$ may not have explicit analytical expressions and will need to be calculated numerically.

In this manuscript, we showed that increasing the quality of the bad patches increases invasion speed. This is in agreement with previous studies showing the importance of the bad patches (habitat matrix) $[45,46]$. For both kernels, increasing the landscape period $L$ reduces the total proportion of good habitat required for an invasion to occur. This is in agreement with the theoretical findings of Shigesada et al (1986) [47] and Kinezaki et al (2010) [48], who found that larger fragmentation scales $L$ reduced the total 
proportion of suitable habitat needed for an invasion to occur in partial differential equation (PDE) models of invasion. Furthermore, we found, with the exception of a small region of parameter space in Figure 5d, that for proportions of good habitat $p$ corresponding to a successful invasion for low landscape periods $L$, increasing the landscape period increases the wave speed $\hat{c}$ for the Laplace kernel, and decreases the wave speed for the Gaussian kernel. It is important and interesting that the choice of kernel affects the relationship between landscape structure and wave-speed, and further work is needed to explore this finding for a range of kernels. Given that increasing good patch size $L_{1}$ increases the wave-speed, and increasing the distance between good patches $L-L_{1}$ decreases the wave-speed, we hypothesise that the effect of increasing the landscape period (i.e. both patch-size and isolation simultaneously) will depend on the relative strengths of the two effects. For the Laplace kernel, the increase in wave-speed due to the increased $L_{1}$ outweighs the decrease due to increased distance between good patches. For the Gaussian kernel, its thinner tail (compared to the Laplace kernel) increases the effect of increased distance between good patches, to the extent that the effect of increased distance outweighs the effect of increased patch-size. In general, therefore, it is important to have a detailed characterisation of the kernel, if we are to determine the effects of spatial heterogeneity on spread.

The dispersal kernel of a species may vary with habitat quality [17]. For example, plant height is affected by habitat quality [49], and mechanistic models (e.g. [50]) demonstrate that seed release height strongly affects the dispersal kernel. Here, we have presented a method for deriving the propagation speeds of populations in an IDE model where the kernel, the population projection matrix and their associated parameters take different values in the good and bad patches, but for simplicity, have looked only at examples where the dispersal kernel and parameters are constant throughout the landscape. Incorporating heterogeneous dispersal could help understand the way in which spatially heterogeneous dispersal affects populations' spreading speeds. We report on this elsewhere.

Here, we have required the ratio of all the demographic rates in the bad patches to the corresponding rates in the good patches to be $\mathrm{O}(\epsilon)$, and have found that the wave-speeds of stage-structured populations with two demographic stages differ from the $\epsilon=0$ case by $\mathrm{O}\left(\epsilon^{2}\right)$. Ecologically, some species may only be affected by patch type at particular points in their life history, e.g. it may be much more difficult for a juvenile to become established outside the good habitat, but once established will exhibit similar demographic rates to established individuals in the good habitat (for example, see [17]). In some of these cases, it may still be possible for our model to work (by weakening Condition V). For example, our analysis would still be valid where the $\mathrm{O}(1)$ rates in the bad patches could be incorporated into the operator $F_{s, 0}$ without affecting the value of the principal eigenvalue $\rho_{s, 0}^{\text {app }}$ (i.e. $F_{s, 0}$ is determined only by behaviour in the good patches). Whether this is possible will depend on which demographic stages disperse and which stages are adversely affected by poor habitat. Weakening this condition would allow us to incorporate a wider range of behaviours 
in the bad patches.

We have considered single species invasions. A natural extension would be to consider multi species invasions, either where multiple co-operative species are invade together, or where an invader out-competes an existing species. The possibility of extending this methodology to multi-species systems depends on whether solutions are propagating into unstable steady states, and on whether the wave-speeds of the solutions are determined by their linearisation around the unstable population state. Multi-species systems differ from single-species systems in that the linearisation $\mathbf{A}$ of the demographic matrix $\mathbf{B}$ is not generally irreducible. Weinberger [41] has shown analytically that systems with reducible matrices in which the population projection matrix is cooperative/order-preserving $(\mathbf{u}(x) \geq \mathbf{v}(x) \Longrightarrow \mathbf{B}(\mathbf{u}(x), x) \mathbf{u}(x) \geq \mathbf{B}(\mathbf{v}(x), x) \mathbf{v}(x))$ or can be transformed to one by a change of variables have linearly determined wave speeds, but this is not applicable in general. Whether the cooperativity requirement can be relaxed to Condition II for reducible matrices requires further investigation.

In conclusion, we have provided analytical approximations to the wave-speeds of solutions to IDEs in periodic landscapes in which the landscape scale is not constrained by the dispersal scale. We have found them to be accurate, highlighting the sensitivity of the relationship between the landscape parameters and the spreading speed with respect to the choice of kernel. Finally, we have shown the approximations to be extendible to different scenarios.

\section{Acknowledgements}

MAG acknowledges the support of a Natural Environment Research Council Doctoral Training Grant [grant number NE/J500045/1].

\section{Appendix A. Full Derivation of the Approximation}

We now provide a full derivation for the approximations given in $\S 2.5$.

\section{Linear Conjecture}

Following Neubert and Caswell (2000) [23], we assume that Condition II (§2.3) is sufficient to guarantee the wave-speeds of solutions to stage-structured IDEs propagating into an unstable zero population state are determined by their linearisation around the zero population state (the wave-speed is linearly determined), and that these conditions extend periodic stage-structured IDEs.

Weinberger (2002) and Lui (1989) rigorously proved linear determinacy for non-stage structured periodic IDEs [42] and multi-species co-operative homogeneous IDEs [51] (mathematically, this includes single-species 
stage structured IDEs) under the conditions in $\S 2.3$, together with the condition that the IDE is orderpreserving, which is guaranteed when the population projection matrix or growth function is order preserving, i.e. for stage structured populations

$$
\mathbf{u}(x) \geq \mathbf{v}(x) \Longrightarrow \mathbf{B}(\mathbf{u}(x), x) \mathbf{u}(x) \geq \mathbf{B}(\mathbf{v}(x), x) \mathbf{v}(x)
$$

where the inequalities are calculated component-wise. The linearisation of (5) is

$$
\mathbf{u}^{t+1}(x)=\int_{-\infty}^{\infty}[\mathbf{K}(x-y, y) \circ \mathbf{A}(y)] \mathbf{u}^{t}(y) \mathrm{d} y
$$

where $\mathbf{A}(y)$ is the value of $\mathbf{B}\left(\mathbf{u}^{t}(y), y\right)$ at the zero population state. This has solutions of the form

$$
\mathrm{e}^{s(c t-x)} \phi_{s}(x)
$$

where $\phi_{s}(x)$ is $L$-periodic [42]. Solutions to (A.1) with compact initial conditions are bounded above by a multiple of a solution of the form (A.2) for every $s>0$. Therefore the wave-form cannot be travelling faster than the infimum of $c(s)$. Furthermore, since the wavefronts of solutions to (5) are observed numerically to become exponential in shape as $t \rightarrow \infty$, the asymptotic invasion speed of solutions to (A.1) with compact initial conditions must attain one of the wavespeeds of these solutions. Therefore, this wavespeed is the infimum of the wavespeeds of the solutions of form (A.2).

Substituting (A.2) into (A.1), we have that for $s>0$,

$$
\mathrm{e}^{s c(s)} \phi_{s}(x)=\int_{-\infty}^{\infty}[\mathbf{K}(x-y, y) \circ \mathbf{A}(y)] \mathrm{e}^{s(x-y)} \phi_{s}(y) \mathrm{d} y
$$

for some $L$-periodic function $\phi_{s}(y)$. For each $s$, this is an eigenvalue problem, with the principal eigenfunction $\phi_{s}(x)$ and eigenvalue $\rho_{s}:=\mathrm{e}^{s c(s)}$ corresponding to the physical solution [23].

Since $\boldsymbol{\phi}_{s}(y), \mathbf{K}(z, y)$ and $\mathbf{A}(y)$ are $L$-periodic in $y$, we will write (A.3) as

$$
\rho_{s} \boldsymbol{\phi}_{s}(x)=\sum_{n \in \mathbb{Z}} \int_{0}^{L}[\mathbf{K}(x-y-n L, y) \circ \mathbf{A}(y)] \mathrm{e}^{s(x-y-n L)} \phi_{s}(y) \mathrm{d} y
$$

\section{Asymptotic Expansion in $\epsilon$}

We now use Conditions IV and V to find an analytic expansion for $\rho_{s}$, by (i) formulating the integral operator in (A.4) as the sum of an $O(1)$ and $O(\epsilon)$ operator, (ii) approximating the unperturbed operator with an analytically tractable one, and (iii) finding asymptotic expansions for the eigenvalues and eigenvectors of the approximation to the operator.

In $\S 2.2$, we chose $\mathbf{B}$ such that its linearisation on $\left[-L_{1} / 2, L-L_{1} / 2\right)$ can be written as 


$$
\mathbf{A}(y)=\mathbf{A}^{\mathcal{G}} \mathcal{I}_{\left[\frac{-L_{1}}{2}, \frac{L_{1}}{2}\right)}(y)+\epsilon \mathbf{A}^{\mathcal{B}} \mathcal{I}_{\left[\frac{L_{1}}{2}, L-\frac{L_{1}}{2}\right)}
$$

where $\mathbf{A}^{\mathcal{G}}=\mathbf{B}^{\mathcal{G}}(\mathbf{0})$ and $\mathbf{A}^{\mathcal{B}}=\mathbf{B}^{\mathcal{B}}(\mathbf{0})$. This allows us to write (A.4) as

$$
\begin{aligned}
\rho_{s} \phi_{s}(x)= & \sum_{n \in \mathbb{Z}}\left(\int_{\frac{-L_{1}}{2}}^{\frac{L_{1}}{2}}\left[\mathbf{K}^{\mathcal{G}}(x-y-n L) \circ \mathbf{A}^{\mathcal{G}}\right] \mathrm{e}^{s(x-y-n L)} \phi_{s}(y) \mathrm{d} y\right. \\
& \left.+\epsilon \int_{\frac{L_{1}}{2}}^{L-\frac{L_{1}}{2}}\left[\mathbf{K}^{\mathcal{B}}(x-y-n L) \circ \mathbf{A}^{\mathcal{B}}\right] \mathrm{e}^{s(x-y-n L)} \phi_{s}(y) \mathrm{d} y\right) \\
= & \left(F_{s, 0} \phi_{s}\right)(x)+\epsilon\left(F_{s, 1} \phi_{s}\right)(x) .
\end{aligned}
$$

To find the approximate value of $\rho_{s}$, we will use a more analytically tractable operator $F_{s, 0}^{\text {app }}$ to approximate the operator $F_{s, 0}$

$$
F_{s, 0} \mathbf{u}(x)=\sum_{n \in \mathbb{Z}} \int_{\frac{-L_{1}}{2}}^{\frac{L_{1}}{2}}\left[\mathbf{K}^{\mathcal{G}}(x-y-n L) \circ \mathbf{A}^{\mathcal{G}}\right] \mathrm{e}^{s(x-y-n L)} \mathbf{u}(y) \mathrm{d} y
$$

By condition IV in $\S 2.3$ we have that the dispersal kernel $K_{i, j}^{\mathcal{G}}(x)$ for individuals transitioning from stage $j$ to stage $i$ is either (i) a Dirac delta function (individuals going from stage $j$ to stage $i$ do not disperse), or (ii) an exponentially bounded (Condition III) dispersal kernel, such that for $|x|>L_{1} / 2$ and $y \in\left[\frac{-L_{1}}{2}, \frac{L_{1}}{2}\right.$ ) we have that $K_{i, j}^{\mathcal{G}}(x-y) \approx K_{i, j}^{\mathcal{G}}(x)$. This means that the integrand of each integral in the sum corresponding to dispersal between a good patch and a bad patch or a different good patch $\left(n \neq 0\right.$ or $\left.|x|>L_{1} / 2\right)$ in (A.5) is approximately constant over $y \in\left[\frac{-L_{1}}{2}, \frac{L_{1}}{2}\right]$. The terms corresponding to dispersal between a good patch and a bad patch or a different good patch $\left(n \neq 0\right.$ or $\left.|x|>L_{1} / 2\right)$, can therefore be approximated by taking the first term in the Taylor expansion of

$$
\left[\mathbf{K}^{\mathcal{G}}(x-y-n L) \circ \mathbf{A}^{\mathcal{G}}\right] \mathrm{e}^{s(x-y-n L)} \mathbf{u}(y)
$$

in $y$. Substituting the $\mathrm{O}(1)$ term into (A.5), we have

$$
\int_{\frac{-L_{1}}{2}}^{\frac{L_{1}}{2}}\left[\mathbf{K}^{\mathcal{G}}(x-y-n L) \circ \mathbf{A}^{\mathcal{G}}\right] \mathrm{e}^{s(x-y-n L)} \mathbf{u}(y) \mathrm{d} y \approx L_{1}\left[\mathbf{K}^{\mathcal{G}}(x-n L) \circ \mathbf{A}^{\mathcal{G}}\right] \mathrm{e}^{s(x-n L)} \mathbf{u}(0) .
$$

where higher order terms, of $\mathrm{O}\left(\left(L_{1} / \alpha\right)^{2}\right)$ (or $\mathrm{O}\left(\left(L_{1} / \sigma\right)^{2}\right)$ ) or higher, are ignored. When $|x| \leq L_{1} / 2$, we find that for the $n=0$ term, the integrand varies more quickly.

To approximate this integral, we consider the volume of propagules remaining in their original good patch (having dispersed from some point $y$ in the patch). This quantity shows little sensitivity to the location of $x$ in the good patch (as $L_{1}$ is small compared to the scale of dispersal), and is equal to the integral of $K_{i, j}^{\mathcal{G}}(x-y)$ over $y \in\left[\frac{-L_{1}}{2}, \frac{L_{1}}{2}\right]$. Due to this lack of sensitivity to $x$, we take the first term in the Taylor expansion of 
(A.6) around $x=0$,

$$
\int_{\frac{-L_{1}}{2}}^{\frac{L_{1}}{2}}\left[\mathbf{K}^{\mathcal{G}}(x-y) \circ \mathbf{A}^{\mathcal{G}}\right] \mathrm{e}^{s(x-y)} \mathbf{u}(y) \mathrm{d} y \approx\left[\int_{\frac{-L_{1}}{2}}^{\frac{L_{1}}{2}} \mathbf{K}^{\mathcal{G}}(y) \mathrm{d} y \circ \mathbf{A}^{\mathcal{G}}\right] \mathbf{u}(0)
$$

with terms of $\mathrm{O}\left(\left(L_{1} / \alpha\right)^{2}\right)$ (or $\left.\mathrm{O}\left(\left(L_{1} / \sigma\right)^{2}\right)\right)$ and higher ignored. Therefore, where the discretised kernel, $\hat{K}_{i, j}^{\mathcal{G}}(n L)$ is either (i) the Kroenecker delta function (corresponding to the integrals of the dirac delta functions), or (ii) an approximate integral of $K_{i, j}^{\mathcal{G}}(z)$ over $\left[x-n L-\frac{L_{1}}{2}, x-n L+\frac{L_{1}}{2}\right]$

$$
\hat{K}_{i, j}^{\mathcal{G}}(x-n L)= \begin{cases}\int_{-L_{1} / 2}^{L_{1} / 2} K_{i, j}^{\mathcal{G}}(y) \mathrm{d} y & \text { for } n=x=0 \\ L_{1} K_{i, j}^{\mathcal{G}}(x-n L) & \text { otherwise }\end{cases}
$$

and $F_{s, 0}$ can be approximated by the operator $F_{s, 0}^{\text {app }}$

$$
F_{s, 0}^{\mathrm{app}} \mathbf{u}(x)=\sum_{n \in \mathbb{Z}}\left[\mathbf{K}_{\text {app }}^{\mathcal{G}}(x-n L) \circ \mathbf{A}^{\mathcal{G}}\right] \mathrm{e}^{s(x-n L)} \mathbf{u}(0) .
$$

where $\mathbf{K}_{\text {app }}^{\mathcal{G}}(z)=\left(\hat{K}_{i, j}^{\mathcal{G}}(z)\right)_{i, j=1}^{N}$. This is the Point-Source approximation, which we justify numerically in $\S 3.1 .4$, and we expect the principal eigenvalue $\rho_{s}^{\text {app }}$ to be a good approximation to $\rho_{s}$ and that

$$
\min _{s>0}\left[\frac{1}{s} \log \left(\rho_{s}^{\mathrm{app}}\right)\right] \approx \min _{t>0}\left[\frac{1}{t} \log \left(\rho_{t}\right)\right] .
$$

The problem now is to find the principal eigenvalue $\rho_{s}^{\text {app }}$ of $F_{s}^{\text {app }}=F_{s, 0}^{\text {app }}+\epsilon F_{s, 1}$ for each $s>0$. To do this, we expand the principal eigenvalue, $\rho_{s}^{\text {app }}$ and eigenfunction $\phi_{s}^{\text {app }}$ of $E_{s}$,

$$
\begin{aligned}
& \rho_{s}^{\mathrm{app}}=\rho_{s, 0}^{\mathrm{app}}+\epsilon \rho_{s, 1}^{\mathrm{app}}+\epsilon^{2} \rho_{s, 2}^{\mathrm{app}}+\cdots \\
& \phi_{s}^{\mathrm{app}}(x)=\phi_{s, 0}^{\mathrm{app}}(x)+\epsilon \boldsymbol{\phi}_{s, 1}^{\mathrm{app}}(x)+\epsilon^{2} \phi_{s, 2}^{\mathrm{app}}(x)+\cdots
\end{aligned}
$$

We will evaluate the coefficients of $\epsilon^{0}$ and $\epsilon$. At $\mathrm{O}(1)$, we have that $\rho_{s, 0}^{\text {app }}$ and $\phi_{s, 0}^{\text {app }}$ are the principal eigenvalue and eigenvector of the unperturbed problem

$$
F_{s, 0}^{\mathrm{app}} \boldsymbol{\phi}_{s, 0}^{\mathrm{app}}=\rho_{s, 0}^{\mathrm{app}} \boldsymbol{\phi}_{s, 0}^{\mathrm{app}}
$$

and at $\mathrm{O}\left(\epsilon^{n}\right)$ (for $n \geq 1$ ), we have

$$
F_{s, 0}^{\mathrm{app}} \boldsymbol{\phi}_{s, n}^{\mathrm{app}}+F_{s, 1} \boldsymbol{\phi}_{s, n-1}^{\mathrm{app}}-\sum_{j=0}^{n} \rho_{s, j}^{\mathrm{app}} \boldsymbol{\phi}_{s, n-j}^{\mathrm{app}}=0
$$


Properties of $F_{s, 0}^{\text {app }}$

Evaluating $\left(F_{s, 0}^{\mathrm{app}} \boldsymbol{\phi}_{s, 0}^{\mathrm{app}}\right)(x)$ at $x=0$ we have that

$$
\left(F_{s, 0}^{\mathrm{app}} \boldsymbol{\phi}_{s, 0}^{\mathrm{app}}\right)(0)=\sum_{n \in \mathbb{Z}}\left[\mathbf{K}_{\mathrm{app}}^{\mathcal{G}}(n L) \circ \mathbf{A}^{\mathcal{G}}\right] \mathrm{e}^{s n L} \boldsymbol{\phi}_{s, 0}^{\mathrm{app}}(0)
$$

and that the principal eigenvalue $\rho_{s, 0}^{\text {app }}$ of $F_{s, 0}^{\text {app }}$ is the principal eigenvalue of

$$
\sum_{n \in \mathbb{Z}}\left[\mathbf{K}_{\mathrm{app}}^{\mathcal{G}}(n L) \circ \mathbf{A}^{\mathcal{G}}\right] \mathrm{e}^{s n L}
$$

This is the result given in (13). The corresponding eigenfunction is given by

$$
\phi_{s, 0}^{\mathrm{app}}(x)=\sum_{n \in \mathbb{Z}}\left[\mathbf{K}_{\mathrm{app}}^{\mathcal{G}}(x-n L) \circ \mathbf{A}^{\mathcal{G}}\right] \mathrm{e}^{s(x-n L)} \boldsymbol{\psi}
$$

where $\boldsymbol{\psi}$ is the principal eigenvector of (A.8). In order to find $\rho_{s, 1}^{\text {app }}$ from (A.7), we will need to consider the adjoint operator $F_{s, 0}^{\text {app* }}$ of $F_{s, 0}^{\text {app }}$, which is defined with the inner product

$$
\langle\mathbf{u} \mid \mathbf{v}\rangle=\int_{0}^{1}[\mathbf{u}(x) \cdot \mathbf{v}(x)] \mathrm{d} x
$$

The adjoint of $F_{s, 0}^{\text {app }}$ in the inner product space $L_{2}\left(\left[\frac{-L_{1}}{2}, L-\frac{L_{1}}{2}\right)\right)$ is

$$
\left(F_{s, 0}^{\mathrm{app} *} \mathbf{u}\right)(x)=\delta(x) \sum_{n \in \mathbb{Z}} \int_{\frac{-L_{1}}{2}}^{L-\frac{L_{1}}{2}}\left[\mathbf{K}_{\mathrm{app}}^{\mathcal{G}}(y-n L) \circ \mathbf{A}^{\mathcal{G}}\right]^{\mathrm{T}} \mathrm{e}^{s(y-n L)} \mathbf{u}(y) \mathrm{d} y .
$$

Putting $\mathbf{u}(x)=\delta(x) \mathbf{v}(x)$, we find that

$$
\left(F_{s, 0}^{\mathrm{app} *} \mathbf{u}\right)(x)=\left(\sum_{n \in \mathbb{Z}}\left[\mathbf{K}_{\text {app }}^{\mathcal{G}}(n L) \circ \mathbf{A}^{\mathcal{G}}\right]^{\mathrm{T}} \mathrm{e}^{s n L}\right) \delta(x) \mathbf{v}(x)
$$

and that the principal eigenvalue of $F_{s, 0}^{\text {app* }}$ is the principal eigenvalue of the transpose of (A.8), which is $\rho_{s}^{\text {app }}$. The corresponding eigenfunction is

$$
\tilde{\phi}_{s, 0}^{\mathrm{app}}(x)=\delta(x) \tilde{\boldsymbol{\psi}}
$$

where $\tilde{\boldsymbol{\psi}}$ is the principal eigenvector of the transpose of (A.8). We can now reconsider (A.7)

$$
\left(F_{s, 0}^{\mathrm{app}}-\rho_{s, 0}^{\mathrm{app}}\right) \boldsymbol{\phi}_{s, n}^{\mathrm{app}}+F_{s, 1} \boldsymbol{\phi}_{s, n-1}^{\mathrm{app}}-\sum_{j=1}^{n} \rho_{s, j}^{\mathrm{app}} \boldsymbol{\phi}_{s, n-j}^{\mathrm{app}}=0
$$

which defines $\phi_{s, n}^{\text {app }}$ and $\rho_{s, n}^{\text {app }}$ in terms of lower order terms. We find $\rho_{s, n}^{\text {app }}$ by taking its inner product with $\tilde{\phi}_{s, 0}^{\text {app }}$ 


$$
\left\langle\tilde{\phi}_{s, 0}^{\mathrm{app}} \mid\left(F_{s, 0}^{\mathrm{app}}-\rho_{s, 0}^{\mathrm{app}}\right) \boldsymbol{\phi}_{s, n}^{\mathrm{app}}\right\rangle+\left\langle\tilde{\boldsymbol{\phi}}_{s, 0}^{\mathrm{app}} \mid F_{s, 1} \phi_{s, n-1}^{\mathrm{app}}\right\rangle-\sum_{j=1}^{n} \rho_{s, j}^{\mathrm{app}}\left\langle\tilde{\boldsymbol{\phi}}_{s, 0}^{\mathrm{app}} \mid \phi_{s, n-j}^{\mathrm{app}}\right\rangle=0
$$

By the properties of the inner product and the adjoint operator $F_{s, 0}^{\text {app* }}$

$$
\left\langle\tilde{\phi}_{s, 0}^{\mathrm{app}} \mid\left(F_{s, 0}^{\mathrm{app}}-\rho_{s, 0}^{\mathrm{app}}\right) \phi_{s, 1}^{\mathrm{app}}\right\rangle=\left\langle\left(F_{s, 0}^{\mathrm{app} *}-\rho_{s, 0}^{\mathrm{app}}\right) \tilde{\boldsymbol{\phi}}_{s, 0}^{\mathrm{app}} \mid \boldsymbol{\phi}_{s, 0}^{\mathrm{app}}\right\rangle=0
$$

Substituting this into (A.12) and rearranging, we have an expression for $\rho_{s, n}^{\mathrm{app}}$ in terms of lower order terms in the asymptotic expansions of $\rho_{s}^{\text {app }}$ and $\phi_{s}^{\text {app }}$,

$$
\begin{aligned}
& \rho_{s, 1}^{\mathrm{app}}=\frac{\left\langle\tilde{\boldsymbol{\phi}}_{s, 0}^{\mathrm{app}} \mid F_{s, 1}^{\mathrm{app}} \boldsymbol{\phi}_{s, 0}^{\mathrm{app}}\right\rangle}{\left\langle\tilde{\boldsymbol{\phi}}_{s, 0}^{\mathrm{app}} \mid \boldsymbol{\phi}_{s, 0}^{\mathrm{app}}\right\rangle} \\
& \rho_{s, n}^{\mathrm{app}}=\frac{\left\langle\tilde{\phi}_{s, 0}^{\mathrm{app}} \mid F_{s, 1}^{\mathrm{app}} \boldsymbol{\phi}_{s, n-1}^{\mathrm{app}}\right\rangle-\sum_{j=1}^{n-1} \rho_{s, j}^{\mathrm{app}}\left\langle\tilde{\phi}_{s, 0}^{\mathrm{app}} \mid \phi_{s, n-j}^{\mathrm{app}}\right\rangle}{\left\langle\tilde{\phi}_{s, 0}^{\mathrm{app}} \mid \phi_{s, 0}^{\mathrm{app}}\right\rangle} \quad \text { for } n>1
\end{aligned}
$$

For $\phi_{s, n}^{\text {app }}$, we must solve (A.11). Since $F_{s, 0}^{\text {app }}-\rho_{s, 0}^{\text {app }}$ has a non-trivial null-space, we have freedom to choose $\phi_{s, n}^{\text {app }}$ such that

$$
\left\langle\tilde{\phi}_{s, 0}^{\mathrm{app}} \mid \phi_{s, n}^{\mathrm{app}}\right\rangle=0
$$

Combining this projection constraint with (A.13), we have that

$$
\rho_{s, n}^{\mathrm{app}}=\frac{\left\langle\tilde{\phi}_{s, 0}^{\mathrm{app}} \mid F_{s, 1}^{\mathrm{app}} \boldsymbol{\phi}_{s, n-1}^{\mathrm{app}}\right\rangle}{\left\langle\tilde{\phi}_{s, 0}^{\mathrm{app}} \mid \phi_{s, 0}^{\mathrm{app}}\right\rangle} \quad \text { for } n \geq 1 .
$$

This is analogous to standard results from matrix perturbation theory $[52,53]$, which for $n=1$ are derived rigorously in [54]. For $\rho_{s, 1}^{\text {app }}$, we use the definitions of $\phi_{s, 0}^{\text {app }}$ and $\tilde{\phi}_{s, 0}^{\text {app }}$ from (A.9) and (A.10), to give us an expression for the numerator and denominator in (A.14) in terms of $\mathbf{K}$ and $\mathbf{B}$,

$$
\begin{aligned}
\left\langle\tilde{\boldsymbol{\phi}}_{s, 0}^{\mathrm{app}}\left|F_{s, 1}^{\mathrm{app}}\right| \boldsymbol{\phi}_{s, 0}^{\mathrm{app}}\right\rangle & =\tilde{\boldsymbol{\psi}} \cdot\left[\sum_{m, n \in \mathbb{Z}} \int_{\frac{-L_{1}}{2}}^{L-\frac{L_{1}}{2}}\left(\mathbf{K}^{\mathcal{B}}(n L-y, y) \circ \mathbf{A}^{\mathcal{B}}(y)\right)\left(\mathbf{K}_{\mathrm{app}}^{\mathcal{G}}(m L+y) \circ \mathbf{A}^{\mathcal{G}}\right) \mathrm{e}^{s(n+m) L} \mathrm{~d} y\right] \boldsymbol{\psi} \\
\left\langle\tilde{\boldsymbol{\phi}}_{s, 0}^{\mathrm{app}} \mid \boldsymbol{\phi}_{s, 0}^{\mathrm{app}}\right\rangle & =\tilde{\boldsymbol{\psi}} \cdot\left[\sum_{n \in \mathbb{Z}}\left(\mathbf{K}_{\mathrm{app}}^{\mathcal{G}}(n L) \circ \mathbf{A}^{\mathcal{G}}\right) \mathrm{e}^{s n L}\right] \boldsymbol{\psi} \\
& =\rho_{s, 0}^{\mathrm{app}} \tilde{\boldsymbol{\psi}} \cdot \boldsymbol{\psi}
\end{aligned}
$$

Substituting these into (A.14) gives us the definition of $\rho_{s, 1}^{\text {app }}$ in (13). 


\section{Appendix B. Numerical Methods}

In $\S 3$ (Examples), we compare our analytical results to simulations of the IDE. We now discuss some of the methods used.

\section{Analytical Approximation}

For exponentially bounded kernels, the terms in (11) and (13) corresponding to large $n, m$ become exponentially small. We have found numerically that the relative errors from taking only the $|n| \leq 20$ terms in (11) and the $|n|,|m| \leq 20$ terms in (13) are less than $10^{-2}$ for both kernels studied (Gaussian and Laplace). Therefore, in calculating our approximations, we use only the $|n|,|m| \leq 20$ terms.

\section{Simulations}

We simulate the IDEs by discretising the population density function and dispersal kernels into domains of non-dimensional length $\approx 0.0015$. Since the kernel in the good patch $\mathbf{K}^{\mathcal{G}}(x-y)$ and the kernel in the bad patch $\mathbf{K}^{\mathcal{B}}(x-y)$ are dependent only on the dispersal distance $x-y$, the RHS of (5) can be written as the sum of two terms, with one referring to dispersal from the good patches, and the other to dispersal from the bad patches. Since the dispersal pattern from each type of patch does not vary within that patch type, the integrals are convolutions of the population density function in that patch type and the dispersal kernel from that patch type. Under the discretisation, the integral convolution of the population density function and dispersal kernel becomes a discrete convolution which we calculate using the Fourier convolution theorem and Fast Fourier Transform (FFT) [55]. We assume the solution will have settled into a travelling wave by $t \sim 50$, we run the simulation for 100 time-steps and use the positions of the wave-front (the maximum of $x$ such that the juvenile stage of the nondimensionalised population density $\left.u_{1}^{t}(x)>\mu\right)$ for $t=75, \ldots, 100$ to find the asymptotic wave-speed by fitting a linear function to the data. Numerically, we find that (after an initial transient) the wave-front maintains the same exponential shape and shifts by a fixed distance. Therefore, the observed wave-speed is independent of the final time choice, and our choice of numerical threshold for invasion $\mu$ which we thereafter take as $\mu=0.0005$. 


\section{Appendix C. Parameter Table}

\begin{tabular}{|c|c|c|}
\hline Parameter & Symbol & Range \\
\hline Time & $t$ & {$\left[\begin{array}{ll}1 & 100\end{array}\right]$} \\
\hline Spatial Variables & $x, y$ & \\
\hline Population density at $x$ at $t$ & $\mathbf{u}^{t}(x)$ & \\
\hline Landscape Period & $L$ & {$\left[\begin{array}{ll}0.25 & 10\end{array}\right]$} \\
\hline Good Patch size & $L_{1}$ & {$\left[\begin{array}{ll}0.01 & 0.2\end{array}\right]$} \\
\hline Proportion of Good Habitat, $p=L_{1} / L$ & $p$ & {$\left[\begin{array}{ll}0.001 & 0.8\end{array}\right]$} \\
\hline Ratio of Demographic Parameters in Good and Bad Patches & $\epsilon$ & $0,0.05,0.25$ \\
\hline Non-stage structured zero-population growth rate in Good patches & $r$ & 10 \\
\hline Gaussian Dispersal Parameter & $\sigma$ & $0.3,1$ \\
\hline Laplace Dispersal Parameter & $\alpha$ & $0.3,1$ \\
\hline Wave-number & $s$ & \\
\hline Wave-speed & $c(s)$ & \\
\hline Principal Eigenvalue of (A.4) & $\rho_{s}$ & \\
\hline Approximation to $\rho_{s}$ using Point-Source Approx. & $\rho_{s}^{\mathrm{app}}$ & \\
\hline$n$-th order term in asymptotic expansion of $\rho_{s}^{\text {app }}$ in $\epsilon$ & $\rho_{s, n}^{\mathrm{app}}$ & \\
\hline Asymptotic wave-speed & $\hat{c}$ & \\
\hline Simulated asymptotic wave-speed & $c^{\mathrm{sim}}$ & \\
\hline Analytical approximation of $\hat{c}$ & $c^{\text {app }}$ & \\
\hline Numerical density threshold for invasion & $\mu$ & 0.0005 \\
\hline (Stage-Structured Example) Birth rate & $\phi$ & 30 \\
\hline (Stage-Structured Example) Juvenile survival Rate & $\nu$ & 0.8 \\
\hline (Stage-Structured Example) Maturation rate & $\gamma$ & 0.625 \\
\hline (Stage-Structured Example) Adult survival Rate & $\theta$ & 0.2 \\
\hline
\end{tabular}

\section{References}

[1] M. Williamson, Invasions, Ecography 22 (1) (1999) 5-12.

[2] P. Pyšek, D. M. Richardson, Invasive species, environmental change and management, and health, Annu. Rev. Env. Resour. 35 (1) (2010) 25-55.

[3] P. M. Vitousek, C. M. D’Antonio, L. L. Loope, R. Westbrooks, et al., Biological invasions as global environmental change, Am. Sci. 84 (5) (1996) 468-478. 
[4] D. Pimentel, R. Zuniga, D. Morrison, Update on the environmental and economic costs associated with alien-invasive species in the united states, Ecol. Econ. 52 (3) (2005) 273-288.

[5] Y. Zhou, M. Kot, Discrete-time growth-dispersal models with shifting species ranges, Theor. Ecol. 4 (1) (2011) 13-25.

[6] J. Bennie, J. A. Hodgson, C. R. Lawson, C. T. R. Holloway, D. B. Roy, T. Brereton, C. D. Thomas, R. J. Wilson, Range expansion through fragmented landscapes under a variable climate, Ecol. Lett. 16 (7) (2013) 921-929.

[7] J. M. Bullock, S. M. White, C. Prudhomme, C. Tansey, R. Perea, D. A. P. Hooftman, Modelling spread of british wind-dispersed plants under future wind speeds in a changing climate, J. Ecol. 100 (1) (2012) $104-115$.

[8] A. W. King, K. A. With, Dispersal success on spatially structured landscapes: when do spatial pattern and dispersal behavior really matter?, Ecol. Model. 147 (1) (2002) 23-39.

[9] D. A. P. Hooftman, J. M. Bullock, Mapping to inform conservation: a case study of changes in seminatural habitats and their connectivity over 70 years, Biol. Conserv. 145 (1) (2012) 30-38.

[10] J. Fischer, D. B. Lindenmayer, Landscape modification and habitat fragmentation: a synthesis, Glob. Ecol. Biogeogr. 16 (3) (2007) 265-280.

[11] M. Marvier, P. Kareiva, M. G. Neubert, Habitat destruction, fragmentation, and disturbance promote invasion by habitat generalists in a multispecies metapopulation, Risk. Anal. 24 (4) (2004) 869-878.

[12] J. M. H. Knops, J. R. Griffin, A. C. Royalty, Introduced and native plants of the hastings reservation, central coastal california: a comparison, Biol. Conserv. 71 (2) (1995) 115-123.

[13] J. A. Hodgson, C. D. Thomas, C. Dytham, J. M. J. Travis, S. J. Cornell, The speed of range shifts in fragmented landscapes, PLoS One 7 (10) (2012) e47141.

[14] M. Renton, N. Shackelford, R. J. Standish, Habitat restoration will help some functional plant types persist under climate change in fragmented landscapes, Glob. Chang. Biol. 18 (6) (2012) 2057-2070.

[15] H. Caswell, Matrix population models: construction, analysis and interpretation, 2nd Edition, Sinauer, Sunderland, Massachusetts, USA, 2000.

[16] M. Kot, W. M. Schaffer, Discrete-time growth-dispersal models, Math. Biosci. 80 (1) (1986) 109-136.

[17] J. M. Bullock, R. F. Pywell, S. J. Coulson-Phillips, Managing plant population spread: prediction and analysis using a simple model, Ecol. Appl. 18 (4) (2008) 945-953. 
[18] M. G. Neubert, I. M. Parker, Projecting rates of spread for invasive species, Risk. Anal. 24 (4) (2004) $817-831$.

[19] S. J. Schreiber, M. E. Ryan, Invasion speeds for structured populations in fluctuating environments, Theor. Ecol. 4 (4) (2011) 423-434.

[20] O. Skarpaas, K. Shea, Dispersal patterns, dispersal mechanisms, and invasion wave speeds for invasive thistles, Am. Nat. 170 (3) (2007) 421-430.

[21] F. Van den Bosch, R. Hengeveld, J. A. J. Metz, Analysing the velocity of animal range expansion, Journal of Biogeography (1992) 135-150.

[22] S. M. White, K. A. J. White, Relating coupled map lattices to integro-difference equations: dispersaldriven instabilities in coupled map lattices, J. Theor. Biol. 235 (4) (2005) 463-475.

[23] M. G. Neubert, H. Caswell, Demography and dispersal: calculation and sensitivity analysis of invasion speed for structured populations, Ecology 81 (6) (2000) 1613-1628.

[24] H. Caswell, R. Lensink, M. G. Neubert, Demography and dispersal: life table response experiments for invasion speed, Ecology 84 (8) (2003) 1968-1978.

[25] R. M. Coutinho, W. A. C. Godoy, R. A. Kraenkel, Integrodifference model for blowfly invasion, Theor. Ecol. 5 (3) (2012) 363-371.

[26] M. Kot, M. A. Lewis, P. van den Driessche, Dispersal data and the spread of invading organisms, Ecology 77 (7) (1996) 2027-2042.

[27] M. Kot, Discrete-time travelling waves: ecological examples, J. Math. Biol. 30 (4) (1992) 413-436.

[28] F. van den Bosch, J. A. J. Metz, O. Diekmann, The velocity of spatial population expansion, J. Math. Biol. 28 (5) (1990) 529-565.

[29] D. Mollison, Dependence of epidemic and population velocities on basic parameters, Math. Biosci. 107 (2) (1991) 255-287.

[30] H. F. Weinberger, Long-time behavior of a class of biological models, SIAM J. Math. Anal. 13 (3) (1982) 353-396.

[31] R. W. Van Kirk, M. A. Lewis, Integrodifference models for persistence in fragmented habitats, B. Math. Biol. 59 (1) (1997) 107-137.

[32] F. Lutscher, M. A. Lewis, Spatially-explicit matrix models, J. Math. Biol. 48 (3) (2004) 293-324. 
[33] S. Dewhirst, F. Lutscher, Dispersal in heterogeneous habitats: thresholds, spatial scales, and approximate rates of spread, Ecology 90 (5) (2009) 1338-1345.

[34] K. Kawasaki, N. Shigesada, An integrodifference model for biological invasions in a periodically fragmented environment, Jpn. J. Ind. Appl. Math. 24 (1) (2007) 3-15.

[35] Y. Samia, F. Lutscher, Coexistence and spread of competitors in heterogeneous landscapes, B. Math. Biol. 72 (8) (2010) 2089-2112.

[36] L. Fahrig, Effects of habitat fragmentation on biodiversity, Annu. Rev. Ecol. Evol. Syst. 34 (2003) $487-515$.

[37] J. T. Curtis, The modification of mid-latitude grasslands and forests by man, In Man's Role in Changing the Face of the Earth, ed. W. L. Thomas (1956) pp. 721-736. University of Chicago Press Chicago, IL.

[38] R. F. Holland, S. K. Jain, Insular biogeography of vernal pools in the central valley of california, Am. Nat. 117 (1) (1981) 24-37.

[39] D. A. Driscoll, S. C. Banks, P. S. Barton, D. B. Lindenmayer, A. L. Smith, Conceptual domain of the matrix in fragmented landscapes, Trends Ecol. Evol. 28 (10) (2013) 605-613.

[40] L. Fahrig, How much habitat is enough?, Biol. Conserv. 100 (1) (2001) 65-74.

[41] H. F. Weinberger, M. A. Lewis, B. Li, Analysis of linear determinacy for spread in cooperative models, J. Math. Biol. 45 (3) (2002) 183-218.

[42] H. F. Weinberger, On spreading speeds and traveling waves for growth and migration models in a periodic habitat, J. Math. Biol. 45 (6) (2002) 511-548.

[43] J. R. Poulsen, C. W. Osenberg, C. J. Clark, D. J. Levey, B. M. Bolker, Plants as reef fish: fitting the functional form of seedling recruitment, Am. Nat. 170 (2) (2007) 167-183.

[44] J. Clobert, M. Baguette, T. G. Benton, J. M. Bullock, Dispersal ecology and evolution, 1st Edition, Oxford University Press, 2012.

[45] L. R. Prugh, K. E. Hodges, A. R. E. Sinclair, J. S. Brashares, Effect of habitat area and isolation on fragmented animal populations, Proc. Natl. Acad. Sci. U.S.A. 105 (52) (2008) 20770-20775.

[46] J. F. Franklin, D. B. Lindenmayer, Importance of matrix habitats in maintaining biological diversity, Proc. Natl. Acad. Sci. U.S.A. 106 (2) (2009) 349-350.

[47] N. Shigesada, K. Kawasaki, E. Teramoto, Traveling periodic waves in heterogeneous environments., Theor. Popul. Biol. 30 (1) (1986) 143-160. 
[48] N. Kinezaki, K. Kawasaki, N. Shigesada, The effect of the spatial configuration of habitat fragmentation on invasive spread, Theor. Popul. Biol. 78 (4) (2010) 298-308.

[49] M. B. Soons, G. W. Heil, Reduced colonization capacity in fragmented populations of wind-dispersed grassland forbs, J. Ecol. 90 (6) (2002) 1033-1043.

[50] G. G. Katul, A. Porporato, R. Nathan, M. Siqueira, M. B. Soons, D. Poggi, H. S. Horn, S. A. Levin, Mechanistic analytical models for long-distance seed dispersal by wind, Am. Nat. 166 (3) (2005) 368-381.

[51] R. Lui, Biological growth and spread modeled by systems of recursions. i. mathematical theory, Math. Biosci. 93 (2) (1989) 269-295.

[52] E. Hinch, Perturbation methods, Cambridge University Press, 1991.

[53] G. H. Golub, C. F. Van Loan, Matrix Computation, 3rd Edition, The Johns Hopkins University Press, 1996.

[54] R. A. Horn, C. R. Johnson, Matrix Analysis, 2nd Edition, Cambridge University Press, 2013.

[55] M. Andersen, Properties of some density-dependent integrodifference equation population models, Math. Biosci. 104 (1) (1991) 135-157. 